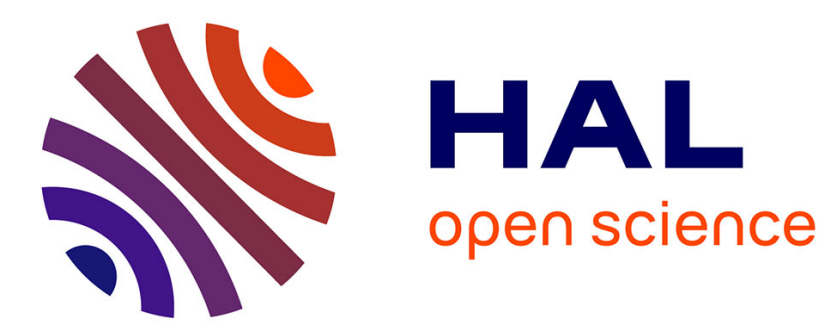

\title{
Two-plane automatic balancing: A symmetry breaking analysis
}

D.J. Rodrigues, A.R. Champneys, M.I. Friswell, R.E. Wilson

\section{To cite this version:}

D.J. Rodrigues, A.R. Champneys, M.I. Friswell, R.E. Wilson. Two-plane automatic balancing: A symmetry breaking analysis. International Journal of Non-Linear Mechanics, 2011, 46 (9), pp.1139. 10.1016/j.ijnonlinmec.2011.04.033 . hal-00784914

\section{HAL Id: hal-00784914 https://hal.science/hal-00784914}

Submitted on 5 Feb 2013

HAL is a multi-disciplinary open access archive for the deposit and dissemination of scientific research documents, whether they are published or not. The documents may come from teaching and research institutions in France or abroad, or from public or private research centers.
L'archive ouverte pluridisciplinaire HAL, est destinée au dépôt et à la diffusion de documents scientifiques de niveau recherche, publiés ou non, émanant des établissements d'enseignement et de recherche français ou étrangers, des laboratoires publics ou privés. 
Two-plane automatic balancing: A symmetry breaking analysis

D.J. Rodrigues, A.R. Champneys, M.I. Friswell, R.E. Wilson

PII: S0020-7462(11)00098-9

DOI: doi:10.1016/j.ijnonlinmec.2011.04.033

Reference: NLM 1869

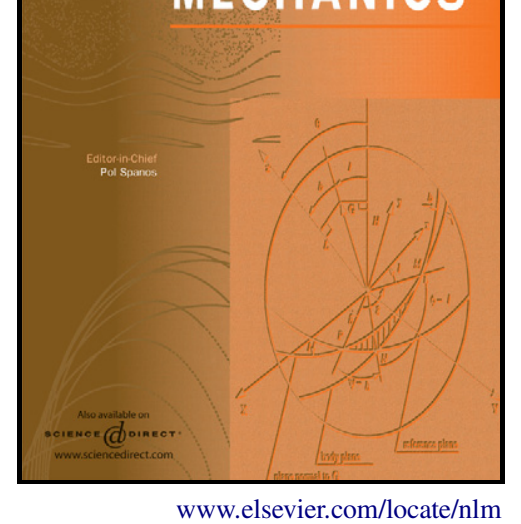

To appear in: International Journal of NonLinear Mechanics

Received date: 25 March 2011

Revised date: 25 March 2011

Accepted date: 26 April 2011

Cite this article as: D.J. Rodrigues, A.R. Champneys, M.I. Friswell and R.E. Wilson, Two-plane automatic balancing: A symmetry breaking analysis, International Journal of Non-Linear Mechanics, doi:10.1016/j.ijnonlinmec.2011.04.033

This is a PDF file of an unedited manuscript that has been accepted for publication. As a service to our customers we are providing this early version of the manuscript. The manuscript will undergo copyediting, typesetting, and review of the resulting galley proof before it is published in its final citable form. Please note that during the production process errors may be discovered which could affect the content, and all legal disclaimers that apply to the journal pertain. 


\title{
Two-plane automatic balancing: a symmetry breaking analysis
}

\author{
D.J. Rodrigues ${ }^{\mathrm{a}}$, A.R. Champneys ${ }^{\mathrm{a}, *}$, M.I. Friswell ${ }^{\mathrm{b}}$ and R.E. Wilson ${ }^{\mathrm{a}}$ \\ ${ }^{a}$ Department of Engineering Mathematics, University of Bristol, Bristol, BS8 1TR, UK \\ ${ }^{b}$ School of Engineering, Swansea University, Swansea, SA2 8PP, UK
}

\begin{abstract}
We present an analysis of a two-plane automatic balancing device for rotating machinery. The mechanism consists of a pair of races that contain balancing balls which move to eliminate imbalance due to rotor eccentricity or principal axis misalignment. A model is developed that includes the effect of support anisotropy and rotor acceleration. The symmetry of the imbalance is considered, and techniques from equivariant bifurcation theory are used to derive a necessary condition for the stability of balanced operation. The unfolding of the solution structure is explored and we investigate mechanical systems in which either the supports or the automatic ball balancer is asymmetric. Here it is shown that, provided the imbalance is small, the balanced state is robust to the considered asymmetries.
\end{abstract}

\section{Introduction}

Mass imbalance is a common cause of vibration in rotating machinery. This occurs when the principal axis of the moment of inertia does not coincide with the axis of rotation. To eliminate the imbalance, mass is usually permanently added or removed from the rotating parts. However, if the mass distribution of the rotor changes then the balancing procedure may need to be repeated. This limitation motivates the study of automatic balancers that use freely moving masses to dynamically compensate for the imbalance [1].

One such device is an automatic ball balancer (ABB), which consists of a series of balls that are free to travel around a race which is set at a fixed distance from the shaft. During operation, the balls tend to find positions such that the principal axis of inertia is repositioned onto the rotational axis. Because the imbalance does not need to be known beforehand, automatic balancers are ideally suited to applications where the imbalance changes with the operating conditions. For example, ABBs are currently used in machine tools, washing machines and optical disk drives [2, 3, 4].

\footnotetext{
${ }^{*}$ Corresponding author. E-mail: A.R.Champneys@bristol.ac.uk
} 
However, given the number of applications for which their use could be envisaged, automatic balancers are still not widely adopted. This is not least because the mechanism is inherently non-linear and displays all the hallmarks of nonlinear dynamics, including bistability and extreme sensitivity to both rotation speed and initial conditions. Therefore, whilst an ABB can compensate for the imbalance for some highly supercritical speeds, it can also make the vibration levels significantly worse during the rotor run-up. Nevertheless, recent advances in the analysis of simple isotropic ABB systems have led to improved predictions of their regions of stability $[5,6]$. However, the set-up on a real machine is usually asymmetric. This paper aims to extend the research by by providing a non-linear bifurcation analysis of a two-plane $\mathrm{ABB}$ for asymmetric configurations that are relevant for the practical implementation of such devices. In particular, we consider the effect of: unequal amounts of imbalance at each race, support anisotropy, balls of differing masses and balancing planes that are not equally spaced from the midspan. In addition we investigate the influence of rotor acceleration for both slow and fast run-ups.

The first academic study of an ABB was carried out by Thearle in 1932 [7], who demonstrated the existence of a stable balanced state at rotation speeds above the first critical speed. There have since been many subsequent analyses of single-plane ABB devices, see for example $[8,9,10,11,12]$ and the references therein. In 1977, Hedaya and Sharp [13] extended the autobalancing concept by proposing a two-plane ABB that can compensate for both unbalanced force and unbalanced moment arising from principal axis misalignment. Later, Sperling et al. [14] used a time averaging approximation to provide a linear stability analysis for such a two-plane device. However, due to the inherent non-linearity of the mechanism, those techniques are not able to accurately predict the stability boundaries of balanced operation. More recently, Rodrigues et al. [6] presented the first non-linear bifurcation analysis of the two-plane ABB. Lagrange's equations and rotating coordinates were used to derive an autonomous set of governing equations. A symmetric set-up was then considered and numerical continuation techniques were employed to compute the stability boundaries of the fully non-linear system with both static and couple imbalances. Moreover, regions of bistability were found in which the balanced state coexists with a desynchronized state that has the balls rotating at a different angular frequency to the rotor.

The rest of this paper is organised as follows. In Section 2 we define the system variables and develop the ABB model so that it includes the effect of support anisotropy and rotor acceleration. In Section 3 the symmetry of the imbalance is considered and methods from equivariant bifurcation theory $[15,16,17]$ are used to construct a reduced model (normal form) that describes the dynamics at the fundamental pitchfork instability. A small couple imbalance is introduced that breaks the symmetry between the two balancing planes and the resulting unfolding of the pitchfork bifurcation is described. The study of the normal form enables us to determine that for stable balanced operation the rotation speed must be higher than both the rigid body critical speeds. However, this condition is not sufficient because the reduced model does not include a description of any secondary oscillatory instabilities (Hopf bifurcations). This 
limitation motivates the analyses of Sections 4 and 5 in which numerical continuation techniques are used to compute the Hopf bifurcation curves that form the stability boundaries of the full system. In particular, Section 4 concerns the cases of asymmetry between the supports and support anisotropy. Section 5 considers the cases where the balls have differing masses and where balancing planes are not equally spaced from the midspan. In both sections it is found that, provided the imbalance is small, the balanced state is robust to the considered asymmetries. Finally, in Section 6 we supplement the results of the steady state analysis by providing simulations of the ABB dynamics that also include the effect of rotor run-up. These simulations show that if the rotor speed is increased too slowly then the balls can 'stall' as the rotor passes through a critical speed. Nevertheless, it is found that this undesirable outcome can be prevented with run-ups that reach supercritical operating speeds more rapidly.

\section{Mathematical Model}

In this section we extend a previously considered ABB model [6] so that it includes effects such as support anisotropy and rotor acceleration. These features can be incorporated by appending the appropriate linear rotor equations with the forcing terms that arise from the motion of the balls.

\subsection{Definition of the variables}

The mechanical device that we wish to model is illustrated in Figure 1, and is based on a rigid rotor that has been fitted with a two-plane automatic balancer $[6,13,14]$. The rotor system in the absence of the balancing balls has mass $M$, principal moments of inertia $\left[J_{t}, J_{t}, J_{p}\right]$, and is mounted on two compliant linear bearings that are located at $S_{1}$ and $S_{2}$. The automatic balancer consists of a pair of races that are set normal to the shaft in two separate planes. Each race contains two balancing balls of mass $m_{k}$, which move through a viscous fluid and are free to travel at a fixed distance $R_{k}$ from the shaft axis. The position of the $k$ th ball is specified by the axial and angular displacements $z_{k}$ and $\alpha_{k}$, which are written with respect to the $C \xi \eta z$ rotor axes.

In order to describe the position and orientation of the rotor, it is helpful to consider the following frames of reference, see Figure 2. We begin with an inertial space frame $O X Y Z$ with origin at $O$ and $Z$-axis oriented along the undeflected bearing centreline. The rotor's lateral motion can be described by introducing a frame $C X^{\prime} Y^{\prime} Z^{\prime}$ with origin at the geometric shaft centre $C$, and axes parallel to those of the $O X Y Z$ space frame. We neglect any motion in the axial direction and so the position vector of the geometric centre $\boldsymbol{r}_{C}$ lies in the $X Y$ plane. The rotor may also perform an out-of-plane tilting motion that can be described as follows: firstly we define intermediate axes $C X^{\prime \prime} Y^{\prime \prime} Z^{\prime \prime}$ that are related to $C X^{\prime} Y^{\prime} Z^{\prime}$ by a rotation of an angle $\phi_{Y}$ about the $Y^{\prime}$ axis, then we rotate $C X^{\prime \prime} Y^{\prime \prime} Z^{\prime \prime}$ about $X^{\prime \prime}$ by an angle $\phi_{X}$, which results in the $C x y z$ axes. Finally, a rotation about the $z$-axis by the spin angle $\theta_{0}$ 


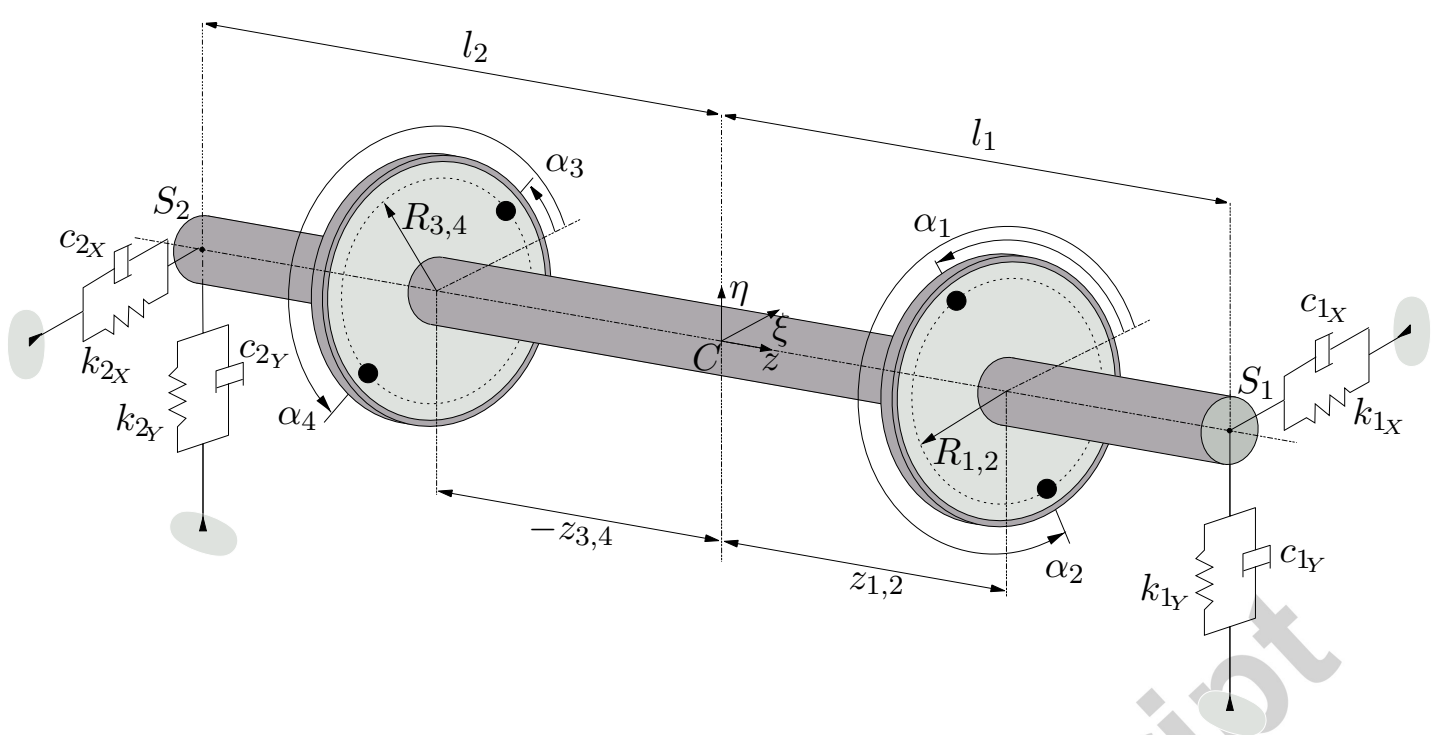

Figure 1: Schematic diagram of a two-plane automatic balancer.

results in the body frame $C \xi \eta z$. These transformations can be combined to give

$$
\xi=\mathrm{R}_{3} \mathrm{R}_{2} \mathrm{R}_{1} \mathbf{X}^{\prime}
$$

where $\boldsymbol{\xi}$ and $\mathbf{X}^{\prime}$ are the column vectors of coordinates in the body and primed axes respectively and the rotation matrices are given by

$$
\mathrm{R}_{1}=\left[\begin{array}{ccc}
\cos \phi_{Y} & 0 & -\sin \phi_{Y} \\
0 & 1 & 0 \\
\sin \phi_{Y} & 0 & \cos \phi_{Y}
\end{array}\right], \quad \mathrm{R}_{2}=\left[\begin{array}{ccc}
1 & 0 & 0 \\
0 & \cos \phi_{X} & \sin \phi_{X} \\
0 & -\sin \phi_{X} & \cos \phi_{X}
\end{array}\right], \quad \mathrm{R}_{3}=\left[\begin{array}{ccc}
\cos \theta_{0} & \sin \theta_{0} & 0 \\
-\sin \theta_{0} & \cos \theta_{0} & 0 \\
0 & 0 & 1
\end{array}\right]
$$

The motion of the rotor can therefore be described by the spin angle $\theta_{0}$ and the complex vibrational coordinate vector

$$
\mathbf{q}=\left[\begin{array}{c}
X+i Y \\
\phi_{Y}-i \phi_{X}
\end{array}\right]
$$

Next, and as shown in Figure 3, small errors in the rotor's mass distribution will cause the body axes $C \xi \eta z$ to differ from the principal axes of the moment of inertia. The eccentricity $\epsilon$, which gives rise to the static imbalance, is defined as the distance between the shaft centre $C$ and the rotor's centre of mass $G$. The constant phase with which the static imbalance leads the $\xi$ axis is denoted by $\beta$. The principal axis $p_{3}$ corresponding to the polar moment of inertia may also be misaligned to the shaft axis by an angle $\chi$, and this results in a couple imbalance. We note that the symmetry of the rotor enables the 


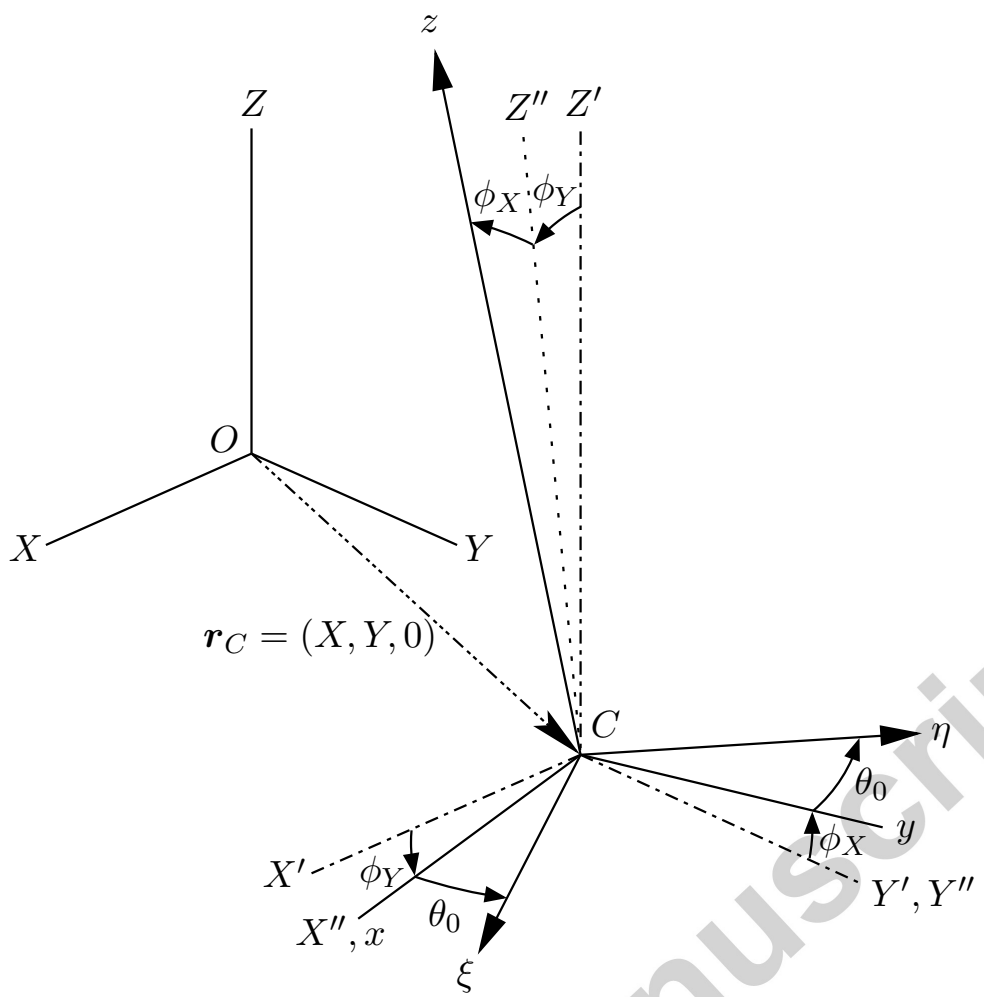

Figure 2: Definition of the coordinate system.

misalignment to be taken about the $\eta$ axis without detracting from the generality of the model.

Finally, the bearings are represented by anisotropic supports which have $X$ and $Y$ as the principal directions of elasticity. To describe this set-up using complex coordinates we introduce the mean and deviatoric stiffness and damping matrices

$$
\begin{aligned}
\mathbf{K}_{\mathrm{m}} & =\frac{1}{2}\left(\mathbf{K}_{X}+\mathbf{K}_{Y}\right), & \mathbf{C}_{\mathrm{m}} & =\frac{1}{2}\left(\mathbf{C}_{X}+\mathbf{C}_{Y}\right), \\
\mathbf{K}_{\mathrm{d}} & =\frac{1}{2}\left(\mathbf{K}_{X}-\mathbf{K}_{Y}\right), & \mathbf{C}_{\mathrm{d}} & =\frac{1}{2}\left(\mathbf{C}_{X}-\mathbf{C}_{Y}\right),
\end{aligned}
$$

where in terms of the individual support parameters

$$
\mathbf{K}_{X}=\left[\begin{array}{cc}
k_{1_{X}}+k_{2_{X}} & k_{1_{X}} l_{1}-k_{2_{X}} l_{2} \\
k_{1_{X}} l_{1}-k_{2_{X}} l_{2} & k_{1_{X}} l_{1}^{2}+k_{2_{X}} l_{2}^{2}
\end{array}\right], \quad \mathbf{C}_{X}=\left[\begin{array}{cc}
c_{1_{X}}+c_{2_{X}} & c_{1_{X}} l_{1}-c_{2_{X}} l_{2} \\
c_{1_{X}} l_{1}-c_{2_{X}} l_{2} & c_{1_{X}} l_{1}^{2}+c_{2_{X}} l_{2}^{2}
\end{array}\right]
$$

and similarly for the $Y$-direction matrices. For further details on complex coordinates and anisotropic rotors, see for example $[18, \S 6]$. 


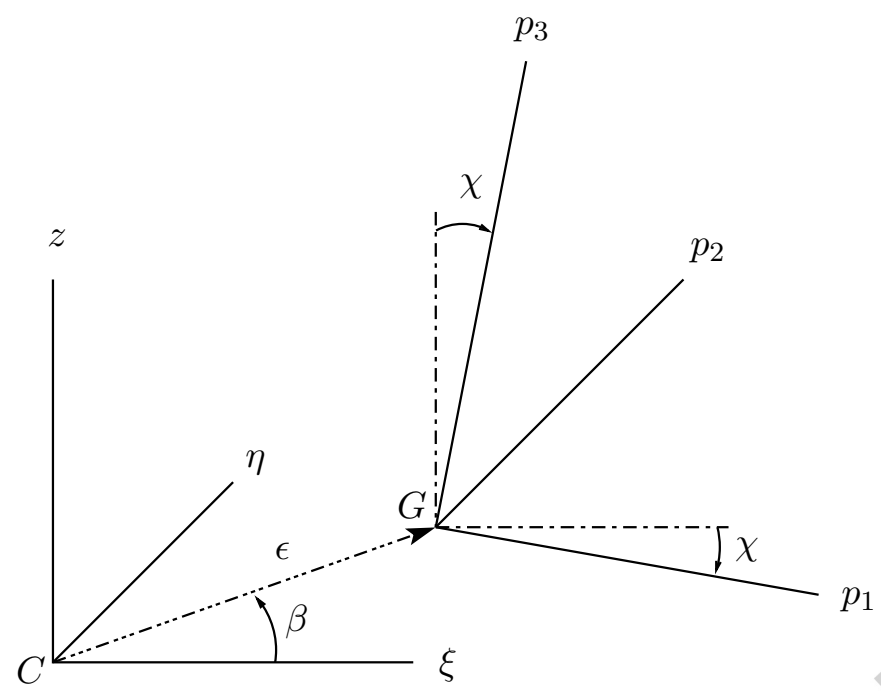

Figure 3: Definition of the imbalance.

\subsection{Equations of motion}

The equations of motion for a two-plane ABB have been derived in $[6,14]$ using Lagrange's equations. We write the system here with respect to the complex vibrational coordinate vector $\mathbf{q}$, which yields

$$
\begin{aligned}
& \mathbf{M} \ddot{\mathbf{q}}+\left(\mathbf{C}_{\mathrm{m}}-i \dot{\theta}_{0} \mathbf{G}\right) \dot{\mathbf{q}}+\mathbf{K}_{\mathrm{m}} \mathbf{q}+\mathbf{C}_{\mathrm{d}} \dot{\overline{\mathbf{q}}}+\mathbf{K}_{\mathrm{d}} \overline{\mathbf{q}} \\
& \quad=\left(\dot{\theta}_{0}^{2}-i \ddot{\theta}_{0}\right) \mathbf{f}_{I} e^{i \theta_{0}}+\sum_{k=1}^{4}\left(\dot{\theta}_{k}^{2}-i \ddot{\theta}_{k}\right) \mathbf{f}_{b_{k}} e^{i \theta_{k}}, \\
& \tilde{J}_{p} \ddot{\theta}_{0}+c_{r} \dot{\theta}_{0}+\sum_{k=1}^{4} c_{b}\left(\dot{\theta}_{0}-\dot{\theta}_{k}\right)+\operatorname{Im}\left(\overline{\mathbf{f}}_{I}^{\mathrm{T}} \ddot{\mathbf{q}} e^{-i \theta_{0}}\right)=\tau\left(\dot{\theta}_{0}\right), \\
& m_{k} R_{k}^{2} \ddot{\theta}_{k}+c_{b}\left(\dot{\theta}_{k}-\dot{\theta}_{0}\right)+\operatorname{Im}\left(\overline{\mathbf{f}}_{b_{k}}^{\mathrm{T}} \ddot{\mathbf{q}} e^{-i \theta_{k}}\right)=0, \quad k=1 \ldots 4 .
\end{aligned}
$$

Here, $\overline{\mathbf{q}}$ is the complex conjugate of $\mathbf{q}$ and $\theta_{k}=\theta_{0}+\alpha_{k}$ is the angular displacement of the $k$ th ball with respect to the non-rotating $C x y z$ axes. The mass and gyroscopic matrices are given respectively by

$$
\mathbf{M}=\left[\begin{array}{cc}
M+\sum_{k} m_{k} & \sum_{k} m_{k} z_{k} \\
\sum_{k} m_{k} z_{k} & J_{t}+\sum_{k} m_{k} z_{k}^{2}
\end{array}\right], \quad \mathbf{G}=\left[\begin{array}{cc}
0 & 0 \\
0 & J_{p}
\end{array}\right],
$$

and the rotor and ball imbalance vectors are given by

$$
\mathbf{f}_{I}=\left[\begin{array}{c}
M \epsilon e^{i \beta} \\
\chi\left(J_{t}-J_{p}\right)
\end{array}\right], \quad \mathbf{f}_{b_{k}}=\left[\begin{array}{c}
m_{k} R_{k} \\
m_{k} R_{k} z_{k}
\end{array}\right]
$$


The torsional behaviour of the rotor is described by equation (3), where $\tilde{J}_{p}=J_{p}+J_{t} \chi^{2}+M \epsilon^{2}$ is the modified polar moment of inertia, $\tau\left(\dot{\theta}_{0}\right)$ is the driving torque generated by the motor and $c_{r}$ is the torque damping. Finally $c_{b}$ is the damping of the balls in the race as they move through the viscous fluid.

We note that by taking $m_{k}=0$ in (2), we recover the equations of motion for a four degree of freedom rotor on anisotropic supports [18]. Also, by setting the tilt angles $\phi_{X}=\phi_{Y} \equiv 0$, the system reduces to the equations of motion for the planar automatic balancer [5]. The form of the governing equations (2)-(4) suggest that automatic balancing can be viewed as a synchronization phenomena of coupled oscillators, see also [19] and [20, $§ 7$. Namely, for smooth operation we require that the ball speeds $\dot{\theta}_{k}$ synchronize with the rotor speed $\dot{\theta}_{0}$ and furthermore that the phases of the balls $\theta_{k}-\theta_{0}$ are such that their forcing cancels out, or at least reduces, the forcing from the rotor imbalance. In addition, the structural similarities of equations (3) and (4) allow us to consider the balls as oscillators that are not acted upon by any external torque, but are driven solely through their coupling with the underlying vibrations of the rotor.

\section{Symmetric isotropic set-up}

In this section we discuss how the symmetry properties of the rotor can affect the bifurcation structure of the ABB. In order to simplify the following analysis we assume a constant rotation speed $\dot{\theta}_{0}=\Omega$, and so equation (3) for the torsional motion of the rotor will be neglected. In addition, we assume that the supports are isotropic so that the deviatoric stiffness and damping matrices are identically zero. In this case the rotor angle $\theta_{0}$ can be eliminated by using the transformation

$$
\mathbf{q}=\mathbf{r} e^{i \theta_{0}}
$$

where

$$
\mathbf{r}=\left[\begin{array}{c}
x+i y \\
\phi_{y}-i \phi_{x}
\end{array}\right],
$$

is the complex vector of the rotating vibrational coordinates. Substituting for $\mathbf{q}$ into equations (2) and (4) then yields

$$
\begin{aligned}
& \mathbf{M \ddot { \mathbf { r } }}+[\mathbf{C}+i \Omega(2 \mathbf{M}-\mathbf{G})] \dot{\mathbf{r}}+\left[\mathbf{K}-\Omega^{2}(\mathbf{M}-\mathbf{G})+i \Omega \mathbf{C}\right] \mathbf{r} \\
& =\Omega^{2} \mathbf{f}_{I}+\sum_{k=1}^{4}\left(\left(\Omega+\dot{\alpha}_{k}\right)^{2}-i \ddot{\alpha}_{k}\right) \mathbf{f}_{b_{k}} e^{i \alpha_{k}} \\
& m_{k} R_{k}^{2} \ddot{\alpha}_{k}+c_{b} \dot{\alpha}_{k}+\operatorname{Im}\left\{\left[\overline{\mathbf{f}}_{b_{k}}^{\mathrm{T}}\left(\ddot{\mathbf{r}}+2 i \Omega \dot{\mathbf{r}}-\Omega^{2} \mathbf{r}\right)\right] e^{-i \alpha_{k}}\right\}=0, \quad k=1 \ldots 4
\end{aligned}
$$


The steady state solutions of the above system are obtained by setting all the time derivatives to zero. Moreover, if we also set the vibrational coordinates $\mathbf{r}=0$, we arrive at the following condition for a balanced steady state

$$
\mathbf{f}_{I}+\sum_{k=1}^{4} \mathbf{f}_{b_{k}} e^{i \alpha_{k}}=0
$$

This equation simply states that the forces and moments acting on the rotor due to the imbalance and the balancing balls must be in equilibrium. We denote the solution of (9) as the balanced state $\mathbf{B}$; it is physically unique and exists provided that the balls have a mass large enough to cope with the imbalance. The balanced state ball positions can be determined in closed form, however the equations are long and so they are not presented here, for further details see [6].

The bifurcation structure of a system is often simplified when there are symmetries in the mechanical set-up. We therefore consider a symmetric isotropic system that has a reflectional symmetry in the transverse plane that includes the centre of rotation $C$, see Figure 1 . We then have the following parameter set

$$
\begin{aligned}
& m_{k}=m, \quad R_{k}=R, \quad z_{1,2}=-z_{3,4}=z, \quad l_{1}=l_{2}=l \\
& k_{1_{X}}=k_{1_{Y}}=k_{2_{X}}=k_{2_{Y}}=k, \quad c_{1_{X}}=c_{1_{Y}}=c_{2_{X}}=c_{2_{Y}}=c
\end{aligned}
$$

and from (1) we also obtain stiffness and damping matrices of the form

$$
\mathbf{K}_{X}=\mathbf{K}_{Y}=\mathbf{K}=\left[\begin{array}{cc}
2 k & 0 \\
0 & 2 k l^{2}
\end{array}\right] \text { and } \mathbf{C}_{X}=\mathbf{C}_{Y}=\mathbf{C}=\left[\begin{array}{cc}
2 c & 0 \\
0 & 2 c l^{2}
\end{array}\right]
$$

In addition, because the interchange of ball races must leave the system invariant, we require that each race contains an equal amount of imbalance. This occurs, for example, when there is a pure static imbalance or a pure couple imbalance.

The physical configurations of the possible ABB steady states in the case of a pure static imbalance are illustrated in Figure 4. The balanced state $\mathbf{B}$ is shown on the left, but there are also various other steady state solutions for which the rotor remains out of balance. The arrangement for $\mathbf{C} \mathbf{3}$ shows coincident balls in the top race while the bottom race balls are split. (Note that the balls are modelled as point masses and the effect of ball interactions are neglected). The corresponding state, where the roles of the races are swapped, is physically equivalent, because the symmetry between the two races is preserved by a static imbalance.

From a practical point of view the most beneficial steady state response is that of the balanced state B which eliminates imbalance due to both rotor eccentricity and principal axis misalignment. Usually 


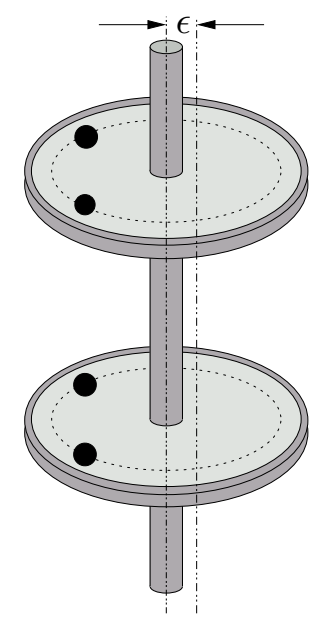

B

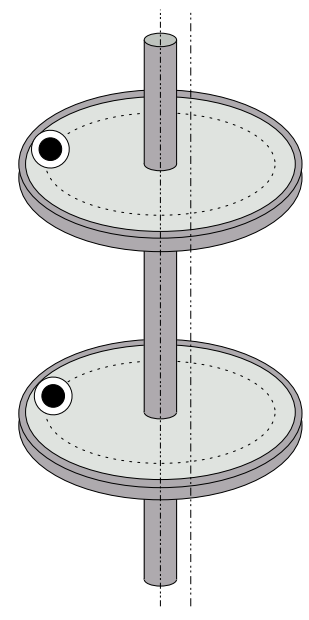

C1

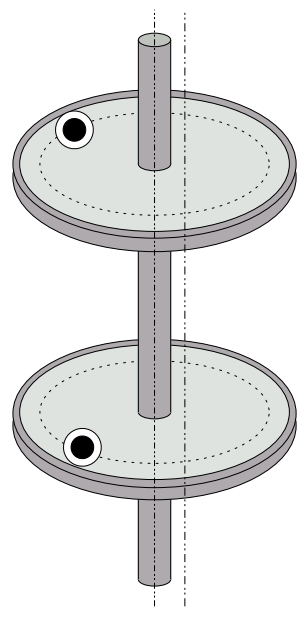

C2

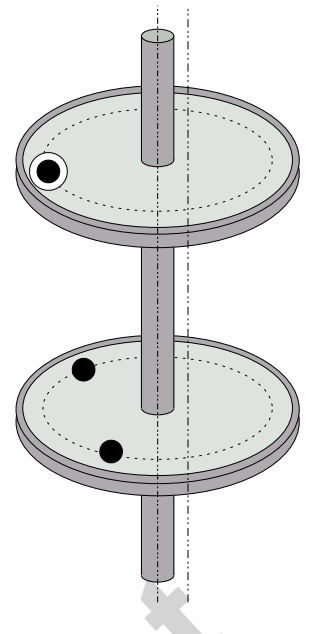

C3

Figure 4: Schematic diagram of the steady states for the case of a static imbalance. White surrounds denote that the two balls in the race are coincident.

one does not know a priori where the balanced state lies. Therefore, in practice the balls are released from opposite sides of the disc so that at least the initial imbalance is not added to.

In order to motivate the following mathematical analysis we first consider a specific ABB system with parameters given by

$$
\begin{aligned}
& M=1, \quad R=1, \quad k=0.5 \\
& J_{t}=3.25, \quad J_{p}=0.5, \quad l=3, \quad z=2 \\
& c=0.01 \quad \text { and } \quad \bar{c}_{b} \equiv \frac{c_{b}}{m}=0.01 .
\end{aligned}
$$

The first three constraints are simply rescalings which make the equations compatible with the nondimensionalised system of [6]. The inertial values are based on a solid cylindrically shaped rotor with a height of six times its radius and the approximate critical speeds for the cylindrical and conical whirls occur respectively at

$$
\Omega_{\mathrm{cyl}}=\sqrt{\frac{2 k}{M}}=1, \quad \text { and } \quad \Omega_{\mathrm{con}}=\sqrt{\frac{2 k l^{2}}{J_{t}-J_{p}}} \simeq 1.81 .
$$

Next, we use the continuation package AUTO [21] to numerically compute the bifurcations and stabilities of the steady states. Figure 5(a) illustrates the bifurcation diagram for the static imbalance case with $\epsilon=0.01$. Here the mass of the balls is the varying parameter, the rotation speed $\Omega=3$ is supercritical to both the cylindrical and conical whirls, and the solution measure is the Euclidean norm of the 
ball angles which is given by

$$
\|\boldsymbol{\alpha}\|=\sqrt{\sum_{k} \alpha_{k}^{2}} \quad \text { where } \quad \boldsymbol{\alpha}=\left(\alpha_{1}, \alpha_{2}, \alpha_{3}, \alpha_{4}\right) .
$$

When the ball mass is too low to fully compensate for the imbalance then $\mathbf{C 1}$ is the only possible steady state solution. Thus the balls are coincident in both races and they reside directly opposite the imbalance. As the ball mass is increased through the critical mass, the $\mathbf{C} 1$ solution branch bifurcates in a degenerate pitchfork and both the balanced state $\mathbf{B}$ and the coincident state $\mathbf{C} 3$ are born. Physically this means that the balls may either split in both races and balance the whole rotor or they may split in only one race which leads to a partial imbalance compensation. For the set of parameters under consideration the balanced state $\mathbf{B}$ is stable at the pitchfork and then undergoes a series of stability changes at three further Hopf bifurcations. However, for other rotation speeds the C3 and B branches may swap stability and so there may be no opportunity for fully balanced operation. In addition, there exists regions for which all of the steady states are unstable. Here, the balls desynchronise with the rotor and this can lead to vibrations that are an order of magnitude worse than if there were no balls present.
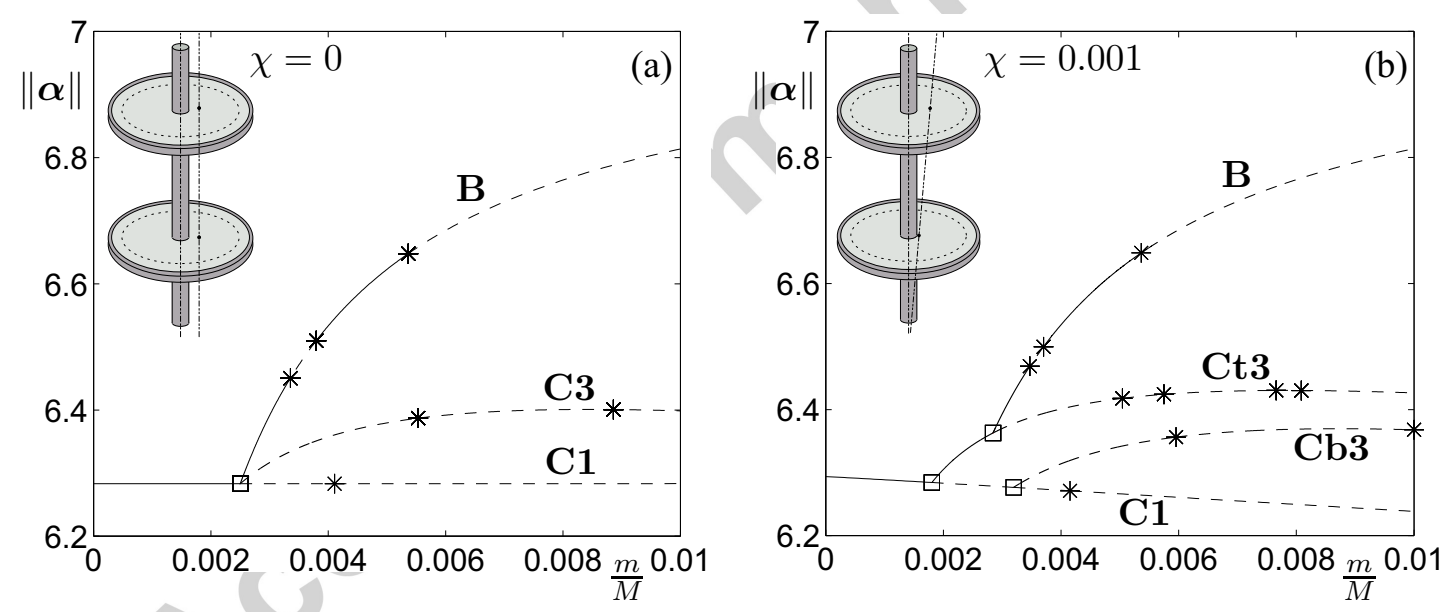

Figure 5: One parameter bifurcation diagrams in $m$ that illustrate the unfolding of the pitchfork bifurcation at which the balanced state is born. Solid and dashed lines represent stable and unstable steady states respectively, $(\square)$ and $(*)$ denote pitchfork and Hopf bifurcations respectively and the labels correspond to the solutions depicted in Figure 4. Panel (a) is for a pure static imbalance with $(\epsilon / R, \beta)=(0.01,0)$, $\Omega=3$ and the other parameters taking values as given in (10). In panel (b) we unfold the degenerate pitchfork by adding a small misalignment $\chi=0.001$ that acts to break the symmetry between the two races.

If a small misalignment $\chi=0.001$ is added to the mass imbalance, we find that the bifurcation unfolds into three non-degenerate pitchforks, panel (b). This unfolding occurs because the symmetry between the two races is broken. When a misalignment is introduced, there is more imbalance at one race (in this case the top one) than at the other. As a consequence, the steady state C3 unfolds into 
two physically different solutions. The configuration with coincident balls in the top race is called Ct3, whereas the corresponding state with the bottom race balls coincident is $\mathbf{C b 3}$.

Although we may intuitively expect the behaviour that is seen in Figure 5, a more detailed investigation into the relative stabilities of the bifurcating branches will require some of the techniques of equivariant bifurcation theory. For further details on this topic see for example [15], [22] and in particu$\operatorname{lar}[16]$.

\subsection{Symmetry identification}

We can recast the equations of motion as a first order system in the usual way by defining a state vector

$$
\boldsymbol{u}=[\mathbf{r}, \boldsymbol{\alpha}, \dot{\mathbf{r}}, \dot{\boldsymbol{\alpha}}]^{\mathrm{T}}
$$

and substituting it component-wise back into (7) and (8). The system then takes the form

$$
\mathcal{A}(\boldsymbol{u}, \mu) \frac{\mathrm{d} \boldsymbol{u}}{\mathrm{d} t}=\mathcal{B}(\boldsymbol{u}, \mu)
$$

where $\mu$ is the bifurcation parameter, $\mathcal{A}$ is a generalised mass matrix and $\mathcal{B}$ is a smooth right-hand-side function.

Let $\gamma$ be a linear transformation that acts on the state variables. For $\gamma$ to be a symmetry of the system, it must transform both the equations and the state variables in the same manner. Considering just the left hand side of (12) we require that

$$
\begin{aligned}
\gamma \mathcal{A}(\boldsymbol{u}, \mu) \frac{\mathrm{d} \boldsymbol{u}}{\mathrm{d} t} & =\mathcal{A}(\gamma \boldsymbol{u}, \mu) \frac{\mathrm{d}}{\mathrm{d} t}(\gamma \boldsymbol{u}) \\
& =\mathcal{A}(\gamma \boldsymbol{u}, \mu) \gamma \frac{\mathrm{d} \boldsymbol{u}}{\mathrm{d} t}
\end{aligned}
$$

where the second equality comes from the fact that $\gamma$ is time independent. Since this condition must hold for all values of $\mathrm{d} \boldsymbol{u} / \mathrm{d} t$, we have that

$$
\gamma \mathcal{A}(\boldsymbol{u}, \mu) \gamma^{-1}=\mathcal{A}(\gamma \boldsymbol{u}, \mu)
$$

Similarly, for the right-hand side of the system we require that

$$
\gamma \mathcal{B}(\boldsymbol{u}, \mu)=\mathcal{B}(\gamma \boldsymbol{u}, \mu)
$$

Together (13) and (14) are the equivariance conditions for the generalised mass matrix $\mathcal{A}$, and the righthand side function $\mathcal{B}$, respectively.

The set of symmetries $\left\{\gamma_{1}, \gamma_{2}, \ldots\right\}$ form a group $\Gamma$ under composition. In order to determine the 
group structure of $\Gamma$, we first need to find all the physical transformations that leave the ABB set-up invariant. Because each ball is identical, the interchange of balls within a race is a symmetry and we denote these operations by

$$
\gamma_{12}=\left(\alpha_{1}, \dot{\alpha}_{1}\right) \leftrightarrow\left(\alpha_{2}, \dot{\alpha}_{2}\right) \quad \text { and } \quad \gamma_{34}=\left(\alpha_{3}, \dot{\alpha}_{3}\right) \leftrightarrow\left(\alpha_{4}, \dot{\alpha}_{4}\right)
$$

If in addition the imbalance is purely static ${ }^{1}$ so that $\chi=0$, then the interchange of races $\gamma_{r}$ is a further symmetry. However, because the switching of balls between races has the effect of reversing the direction of their moment terms, we must also change the sign of the angular state variables in order to keep the system invariant. Thus we have the symmetry

$$
\gamma_{r}=\left(\alpha_{1}, \alpha_{2}, \phi_{x}, \phi_{y}, \dot{\alpha}_{1}, \dot{\alpha}_{2}, \dot{\phi}_{x}, \dot{\phi}_{y}\right) \leftrightarrow\left(\alpha_{3}, \alpha_{4},-\phi_{x},-\phi_{y}, \dot{\alpha}_{3}, \dot{\alpha}_{4},-\dot{\phi}_{x},-\dot{\phi}_{y}\right)
$$

The transformations $\gamma_{12}, \gamma_{34}$ and $\gamma_{r}$ satisfy the equivariance conditions (13) and (14) and together they generate the symmetry group $\Gamma$.

Next, in order to determine the structure of $\Gamma$ we consider the geometric realisation that is shown in Figure 6. By identifying the corner labels of a square with the ball indices, the group elements $\gamma_{12}, \gamma_{34}$ and $\gamma_{r}$ can be viewed as the indicated reflections. In addition, $\gamma_{12} \gamma_{r}$ is a rotation through an angle of $\pi / 2$ and so the full symmetry group of the square $\mathcal{D}_{4}$ can be generated. Thus we have the isomorphism $\Gamma \cong \mathcal{D}_{4}$.
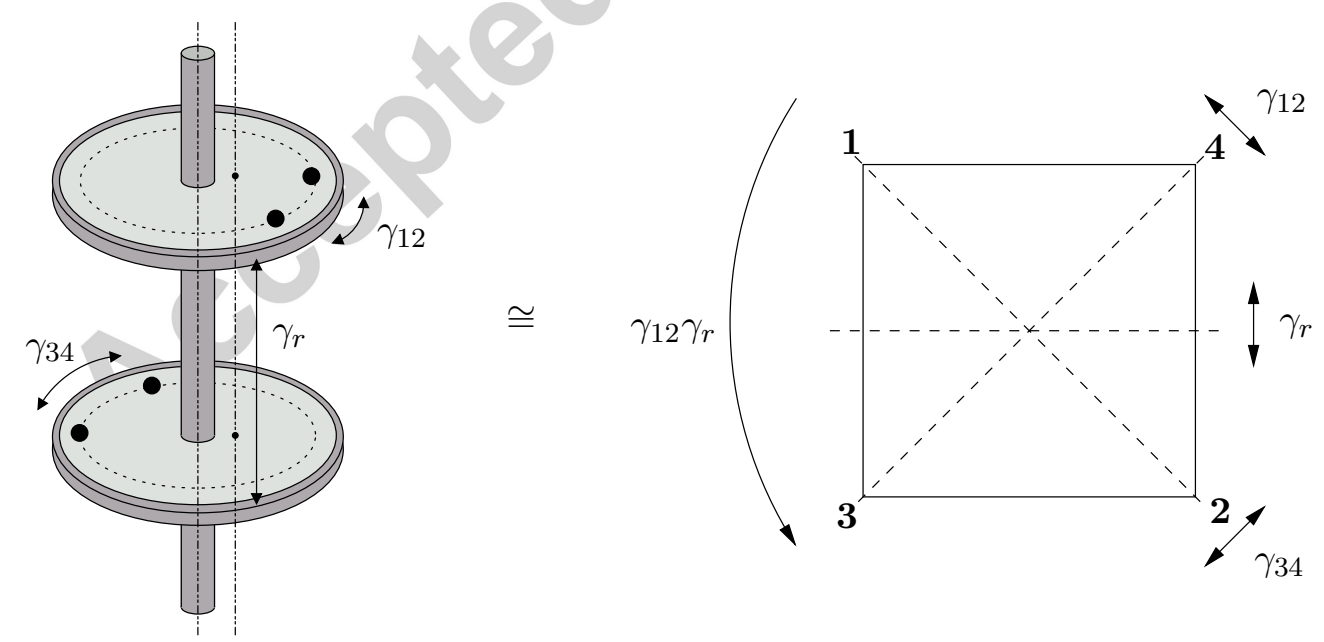

Figure 6: Physical and abstract realisations of the symmetry group that illustrates the isomorphism $\Gamma \cong \mathcal{D}_{4}$ for the case of a static imbalance. Here the corner labels of the square represent the indices of the balls.

\footnotetext{
${ }^{1} \mathrm{~A}$ sufficient condition; the necessary condition is that there is an equal amount of imbalance at each race.
} 


\subsection{Pitchfork bifurcation with $\mathcal{D}_{4}$ symmetry}

Now that we have found the relevant symmetry group for our system, we may characterise the resulting solutions by employing some methods from equivariant bifurcation theory. The properties of steady state bifurcations with $\mathcal{D}_{4}$ symmetry are well known and we shall adapt the treatment that is given in $[16, \S 4]$.

\subsubsection{The normal form for a $\mathcal{D}_{4}$-equivariant pitchfork bifurcation}

From the equivariance conditions (13) and (14) we can deduce that the normal form for a pitchfork bifurcation in a system with this representation of $\mathcal{D}_{4}$ symmetry can be written in the form $[16,(4.72)]$

$$
\left(\begin{array}{c}
\dot{v}_{1} \\
\dot{v}_{2}
\end{array}\right)=\mu\left(\begin{array}{c}
v_{1} \\
v_{2}
\end{array}\right)-a_{1}\left(\begin{array}{c}
v_{1}^{3} \\
v_{2}^{3}
\end{array}\right)-a_{2}\left(\begin{array}{c}
v_{2}^{2} v_{1} \\
v_{1}^{2} v_{2}
\end{array}\right) .
$$

Here, $a_{1}$ and $a_{2}$ are real constants, $\mu$ is the bifurcation parameter and $v_{1}$ and $v_{2}$ correspond to the amplitudes of the eigenvectors that span the centre eigenspace. We can determine from the linearisation of the $\mathrm{ABB}$ system at the $\mathcal{D}_{4}$-equivariant pitchfork bifurcation that the eigenvalues with zero real part have eigenvectors corresponding to the directions of the deviatoric ball angles

$$
\hat{\alpha}_{12}=\frac{\alpha_{1}-\alpha_{2}}{2} \quad \text { and } \quad \hat{\alpha}_{34}=\frac{\alpha_{3}-\alpha_{4}}{2}
$$

We can therefore identify the normal form variables $v_{1}$ and $v_{2}$ with $\hat{\alpha}_{12}$ and $\hat{\alpha}_{34}$.

Next, the bifurcation parameter is the mass of the balls $m$, and the bifurcation occurs when $m=m_{c}$ at which point the balls have enough mass to completely compensate for the imbalance. Therefore, in order to shift the bifurcation to zero we define

$$
\mu=m-m_{c}
$$

where

$$
m_{c}=\frac{M \epsilon}{4 R}
$$

is the critical ball mass for a static imbalance. The normal form (17) can now be rewritten in the notation of the ABB system as

$$
\left(\begin{array}{l}
\mathrm{d} \hat{\alpha}_{12} / \mathrm{d} t \\
\mathrm{~d} \hat{\alpha}_{34} / \mathrm{d} t
\end{array}\right)=\mu\left(\begin{array}{c}
\hat{\alpha}_{12} \\
\hat{\alpha}_{34}
\end{array}\right)-a_{1}\left(\begin{array}{c}
\hat{\alpha}_{12}^{3} \\
\hat{\alpha}_{34}^{3}
\end{array}\right)-a_{2}\left(\begin{array}{c}
\hat{\alpha}_{34}^{2} \hat{\alpha}_{12} \\
\hat{\alpha}_{12}^{2} \hat{\alpha}_{34}
\end{array}\right)
$$


There are two non-trivial fixed point solutions for this reduced system. The first is given by

$$
(\hat{\alpha}, \hat{\alpha}) \quad \text { with } \quad \hat{\alpha}^{2}=\mu /\left(a_{1}+a_{2}\right) .
$$

Here the balls are split evenly in both races and therefore this solution can be identified with the balanced state $\mathbf{B}$. The second solution is of the type

$$
(0, \hat{\alpha}) \quad \text { with } \quad \hat{\alpha}^{2}=\mu / a_{1} .
$$

In this case the balls are split in one of the races but remain coincident in the other, hence this solution can be identified with the state C3.

\subsubsection{Determination of the coefficients $a_{1}$ and $a_{2}$ in terms of the ABB parameters}

In the next part of this procedure we aim to find the coefficients $a_{1}$ and $a_{2}$ of the normal form (20) in terms of the ABB system parameters. One could use an explicit centre manifold reduction, however, this procedure is technically cumbersome. A simpler approach is to compute the best quadratic approximations to the bifurcation branches B and C3 from the original system, and then compare these results with the corresponding solutions (21) and (22) that were obtained from the normal form.

In the case of a static imbalance, we have from the solution to (9) for the balanced state $\mathbf{B}$

$$
\hat{\alpha}_{12}=\hat{\alpha}_{34}=\arccos \left(\frac{M \epsilon}{4 m R}\right) .
$$

Rearranging and expanding as a Taylor series for small angles we obtain

$$
\begin{aligned}
m & =\frac{M \epsilon}{4 R} \sec \left(\hat{\alpha}_{12}\right) \\
& =\frac{M \epsilon}{4 R}+\frac{M \epsilon}{8 R} \hat{\alpha}_{12}^{2}+\mathcal{O}(4),
\end{aligned}
$$

and recalling (19) we have that

$$
\mu \simeq \frac{M \epsilon}{8 R} \hat{\alpha}_{12}^{2}
$$

Thus, by comparing coefficients with the balanced state solution type (21) of the normal form we have

$$
a_{1}+a_{2}=\frac{M \epsilon}{8 R}
$$

We shall now carry out the same procedure for the coincident state C3. In this case we consider the 
equation for the balls (8), which upon setting the time derivatives to zero, yields

$$
\operatorname{Im}\left\{\left(\overline{\mathbf{f}}_{b_{k}}^{\mathrm{T}} \mathbf{r}\right) e^{-i \alpha_{k}}\right\}=0, \quad k=1 \ldots 4
$$

This can be expanded and rearranged to give

$$
\left(x+z_{k} \phi_{y}\right) \sin \alpha_{k}=\left(y-z_{k} \phi_{x}\right) \cos \alpha_{k}, \quad k=1 \ldots 4, \quad \text { with } \quad z_{1,2}=-z_{3,4}=z .
$$

Here $x+z_{k} \phi_{y}$ and $y-z_{k} \phi_{x}$, can be recognised as the $x$ and $y$ deflections of the race centres. For the solution type $\mathbf{C} 3$ we require that $\left(x+z_{k} \phi_{y}, y-z_{k} \phi_{x}\right)=\mathbf{0}$ is satisfied by one of the two races, so that its centre stays fixed at the undeflected position. Without loss of generality we assume that this occurs for the bottom race, that is to say for $z_{3,4}=-z$. We then have $\left(x-z \phi_{y}, y+z \phi_{x}\right)=\mathbf{0}$ so that

$$
\phi_{y}=\frac{x}{z}, \quad \phi_{x}=-\frac{y}{z}
$$

The above relations allow the elimination of the tilt variables $\phi_{x}$ and $\phi_{y}$ from the steady state equations. Also, the balls in the top race are coincident $\alpha_{1}=\alpha_{2}$ so that $\hat{\alpha}_{12}=0$ and from (25) and (26) we obtain

$$
\begin{aligned}
\tan \alpha_{1} & =\left(\frac{y-z \phi_{x}}{x+z \phi_{y}}\right) \\
& =\left(\frac{y}{x}\right)
\end{aligned}
$$

Next we consider the steady state equations for the vibrational coordinates. By setting all time derivatives of the system (7) to zero we arrive at

$$
\left[\mathbf{K}-\Omega^{2}(\mathbf{M}-\mathbf{G})+i \Omega \mathbf{C}\right] \mathbf{r}=\Omega^{2} \mathbf{f}_{I}+\Omega^{2} \sum_{k=1}^{4} \mathbf{f}_{b_{k}} e^{i \alpha_{k}}
$$

By considering the real and imaginary components separately this equation can be expanded to give

$$
\begin{aligned}
& {\left[\begin{array}{cc}
\mathbf{K}-\Omega^{2}(\mathbf{M}-\mathbf{G}) & -\Omega \mathbf{C} \\
\Omega \mathbf{C} & \mathbf{K}-\Omega^{2}(\mathbf{M}-\mathbf{G})
\end{array}\right] \mathbf{x}} \\
& =\Omega^{2}\left[\begin{array}{c}
M \epsilon \\
0 \\
0 \\
0
\end{array}\right]+2 m R \Omega^{2}\left[\begin{array}{c}
\cos \hat{\alpha}_{12} \cos \bar{\alpha}_{12}+\cos \hat{\alpha}_{34} \cos \bar{\alpha}_{34} \\
z\left(\cos \hat{\alpha}_{12} \cos \bar{\alpha}_{12}-\cos \hat{\alpha}_{34} \cos \bar{\alpha}_{34}\right) \\
\cos \hat{\alpha}_{12} \sin \bar{\alpha}_{12}+\cos \hat{\alpha}_{34} \sin \bar{\alpha}_{34} \\
z\left(\cos \hat{\alpha}_{12} \sin \bar{\alpha}_{12}+\cos \hat{\alpha}_{34} \sin \bar{\alpha}_{34}\right)
\end{array}\right] .
\end{aligned}
$$


Here

$$
\mathbf{x}=\left[\operatorname{Re}(\mathbf{r})^{\mathrm{T}}, \operatorname{Im}(\mathbf{r})^{\mathrm{T}}\right]^{\mathrm{T}}=\left[x, \phi_{y}, y,-\phi_{x}\right]^{\mathrm{T}}
$$

and the contributions from the balls have also been rewritten in terms of the mean and deviatoric angular displacements, which are $\bar{\alpha}_{12}, \bar{\alpha}_{34}$ and $\hat{\alpha}_{12}, \hat{\alpha}_{34}$ respectively. It is not possible to find a closed form solution to (28), however, if we consider the case with no damping $\mathbf{C}=\mathbf{0}$ then the equations simplify due to the decoupling between the $x$ and $y$ directions. As a consequence, we find that part of the physically realisable solution is given by

$$
y=0 \quad \text { and } \quad \bar{\alpha}_{12}=\bar{\alpha}_{34}=\pi
$$

Thus summarising from (26) onwards, for the case with no damping we have a C3 solution type of the form

$$
\mathbf{x}=\left[x, \phi_{y}, y,-\phi_{x}\right]^{\mathrm{T}}=[x, x / z, 0,0]^{\mathrm{T}} \quad \text { and } \quad\left[\bar{\alpha}_{12}, \hat{\alpha}_{12}, \bar{\alpha}_{34}, \hat{\alpha}_{34}\right]^{\mathrm{T}}=\left[\pi, 0, \pi, \hat{\alpha}_{34}\right]^{\mathrm{T}}
$$

Substituting this solution back into (28), we find that the bottom two rows are identically zero, and, from expanding the top two rows we obtain

$$
\begin{aligned}
& \left(2 k-\Omega^{2}(M+4 m)\right) x=\Omega^{2}\left(M \epsilon-2 m R\left(\cos \hat{\alpha}_{34}+1\right)\right), \\
& \left(2 k l^{2}-\Omega^{2}\left(J_{t}+4 m z^{2}-J_{p}\right)\right)\left(\frac{x}{z}\right)=2 m R \Omega^{2} z\left(\cos \hat{\alpha}_{34}-1\right) .
\end{aligned}
$$

Then by eliminating $x$ and rearranging for $m R$ we get

$$
m R=\frac{M \epsilon}{2}\left[(\kappa+1) \cos \hat{\alpha}_{34}+(1-\kappa)\right]^{-1},
$$

where

$$
\begin{aligned}
\kappa= & \frac{z^{2}\left(2 k-(M+4 m) \Omega^{2}\right)}{2 k l^{2}-\Omega^{2}\left(J_{t}+4 m z^{2}-J_{p}\right)} \\
= & \frac{z^{2}\left(1-\left(\frac{\Omega}{\Omega_{\mathrm{cyl}}}\right)^{2}\right)}{l^{2}\left(1-\left(\frac{\Omega}{\Omega_{\mathrm{con}}}\right)^{2}\right)}
\end{aligned}
$$


with

$$
\Omega_{\mathrm{cyl}}=\sqrt{\frac{2 k}{M+4 m}} \text { and } \Omega_{\mathrm{con}}=\sqrt{\frac{2 k l^{2}}{J_{t}+4 m z^{2}-J_{p}}} .
$$

The above expressions can be recognised as the critical frequencies for the cylindrical and conical whirls respectively, which are modified to include the contribution from the balls. Next we expand (31) as a Taylor series for small angles to give

$$
m=\frac{M \epsilon}{4 R}+\frac{M \epsilon(1+\kappa)}{16 R} \hat{\alpha}_{34}^{2}+\mathcal{O}(4)
$$

so that

$$
\mu \simeq \frac{M \epsilon(1+\kappa)}{16 R} \hat{\alpha}_{34}^{2}
$$

By comparison with the coefficient of the C3 solution type (22) of the normal form we may identify

$$
a_{1}=\frac{M \epsilon(1+\kappa)}{16 R}
$$

Thus from (23) we finally obtain

$$
a_{1}=\frac{M \epsilon(1+\kappa)}{16 R} \quad \text { and } \quad a_{2}=\frac{M \epsilon(1-\kappa)}{16 R} .
$$

It is clear from (32) that when $\Omega$ is above or below both the critical speeds then $\kappa>0$, and so $a_{1}>a_{2}$. Conversely, when $\Omega$ lies in between the two critical speeds then $\kappa<0$ and so $a_{2}>a_{1}$, see also Figure 7. In summary, we have the conditions

$$
\begin{array}{ll}
a_{1}>a_{2} & \text { for } \Omega<\Omega_{1} \text { or } \Omega>\Omega_{2}, \\
a_{2}>a_{1} & \text { for } \Omega_{1}<\Omega<\Omega_{2} .
\end{array}
$$

Here, $\Omega_{1}$ and $\Omega_{2}$ are the first and second critical speeds respectively, and as we shall discuss next, the comparative sizes of $a_{1}$ and $a_{2}$ will determine the relative stabilities of the bifurcating branches.

\subsubsection{Stability of the bifurcation branches}

At this point, we remind the reader that the following stability analysis is only valid for the dynamics on the centre manifold of the $\mathcal{D}_{4}$-equivariant pitchfork, that is to say, for the reduced system of (20). In the complete system, the trivial branch $\mathbf{C 1}$ may be unstable due to additional pitchfork or Hopf bifurcations, see for example Figure 5. Nevertheless, a stable B type solution in the normal form is a prerequisite for stability of the balanced state in the full model. 

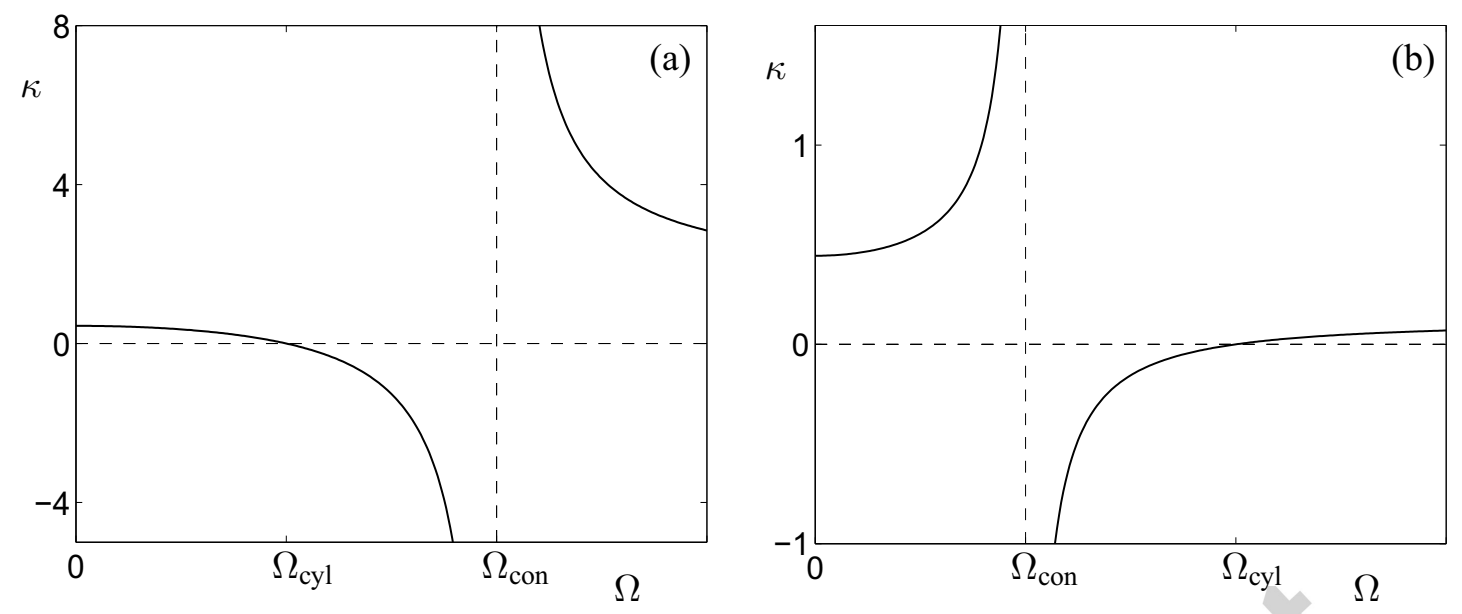

Figure 7: Plots of $\kappa(\Omega)$ as given by equation (32). Panel (a) is for the case with $\Omega_{\text {con }}>\Omega_{\text {cyl }}$, whereas panel (b) is for a set-up with $\Omega_{\text {cyl }}>\Omega_{\text {con }}$. In both cases $\kappa$ is negative in between the critical speeds and is positive otherwise.

The eigenvalues of the Jacobian matrix can be used to find the stability of the bifurcation branches. From the right hand side of the normal form (20) we can compute

$$
\mathrm{D} f=\frac{\mathrm{d} f_{i}}{\mathrm{~d} \hat{\alpha}_{j}}=\left(\begin{array}{cc}
\mu-3 a_{1} \hat{\alpha}_{12}^{2}-a_{2} \hat{\alpha}_{34}^{2} & -2 a_{2} \hat{\alpha}_{12} \hat{\alpha}_{34} \\
-2 a_{2} \hat{\alpha}_{12} \hat{\alpha}_{34} & \mu-3 a_{1} \hat{\alpha}_{34}^{2}-a_{2} \hat{\alpha}_{12}^{2}
\end{array}\right)
$$

Evaluating this at the $\mathbf{C} 3$ type solution with $\hat{\alpha}_{12}=0, \hat{\alpha}_{34}^{2}=\mu / a_{1}$, gives

$$
\mathrm{D} f=\left(\begin{array}{cc}
\mu\left(1-a_{2} / a_{1}\right) & 0 \\
0 & -2 \mu
\end{array}\right)
$$

and so this fixed point is stable if both $\mu>0$ and $a_{2}>a_{1}$. Hence, from condition (35) we require that $\Omega_{1}<\Omega<\Omega_{2}$. Next, if we evaluate the Jacobian at the $\mathbf{B}$ type balanced solution with $\hat{\alpha}_{12}=\hat{\alpha}_{34}$ and $\hat{\alpha}_{12}^{2}=\mu /\left(a_{1}+a_{2}\right)$, we get

$$
\mathrm{D} f=\left(\begin{array}{ll}
-2 a_{1} \mu /\left(a_{1}+a_{2}\right) & -2 a_{2} \mu /\left(a_{1}+a_{2}\right) \\
-2 a_{2} \mu /\left(a_{1}+a_{2}\right) & -2 a_{1} \mu /\left(a_{1}+a_{2}\right)
\end{array}\right)
$$

It is easy to check that this matrix has eigenvalues $-2 \mu$ and $-2 \mu\left(a_{1}-a_{2}\right) /\left(a_{1}+a_{2}\right)$ with eigenvectors $(1,1)$ and $(1,-1)$ respectively. Therefore, the solution is stable if both $\mu>0$ and $a_{1}>a_{2}$. Thus, again from (35), we require that either $\Omega<\Omega_{1}$ or $\Omega>\Omega_{2}$. Finally, the trivial solution $\mathbf{C} 1$ with $\hat{\alpha}_{12}=\hat{\alpha}_{34}=0$ is stable if $\mu<0$ and unstable for $\mu>0$.

The bifurcation diagrams of the $\mathcal{D}_{4}$-equivariant pitchfork are illustrated for the various regions of the $\left(a_{1}, a_{2}\right)$ plane in Figure 8. The condition $a_{1}+a_{2}=M \epsilon / 8 R$, from (23), implies that the particular 


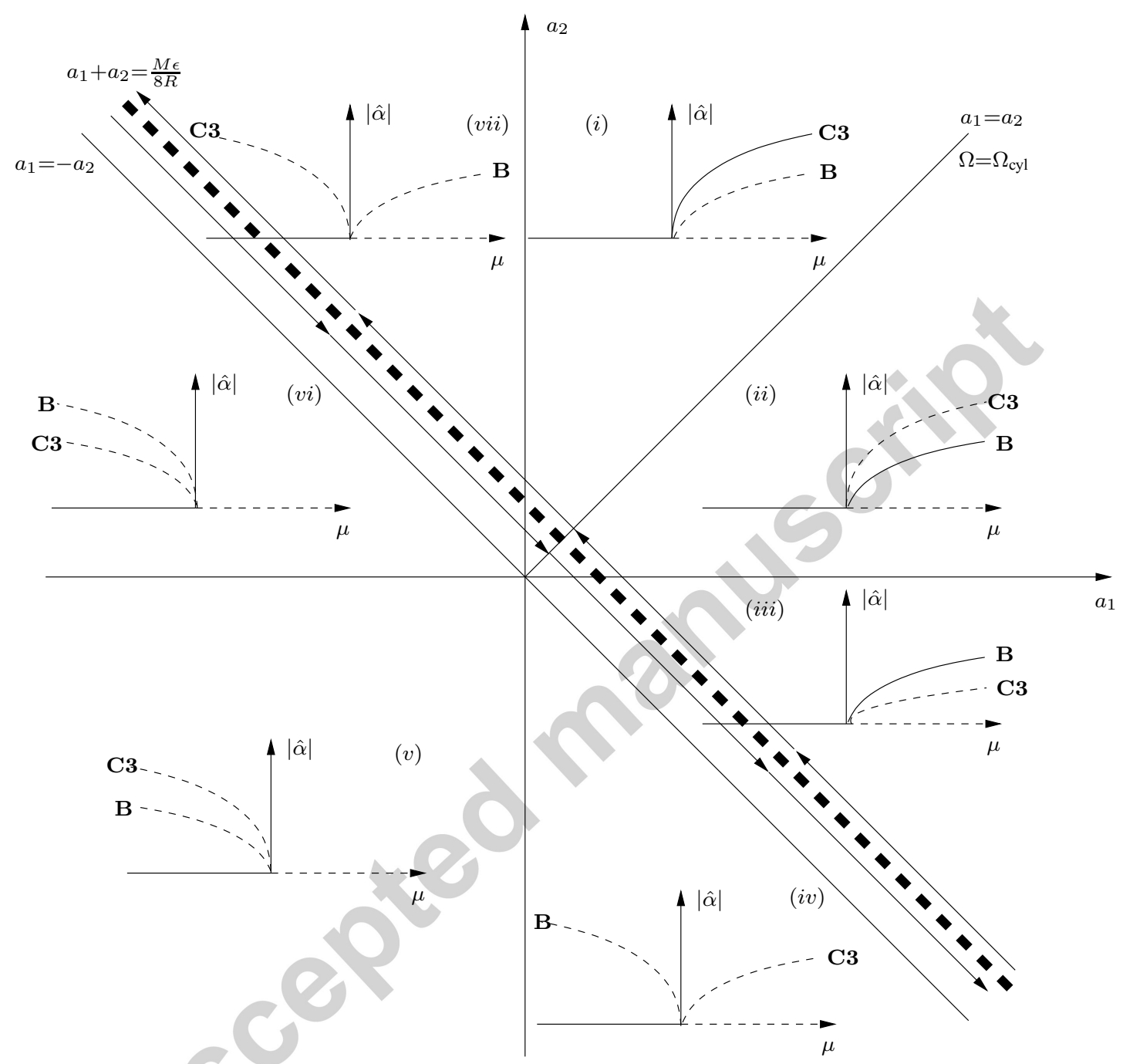

Figure 8: Bifurcation diagrams for the normal form of the $\mathcal{D}_{4}$-equivariant pitchfork bifurcation for various values of the coefficients $a_{1}$ and $a_{2}$ in equation (20). Solid lines represent stable solutions and dashed lines unstable solutions. Branches of the balanced state $\mathbf{B}$ are of the type $(\hat{\alpha}, \hat{\alpha})$ where the balls are split evenly in both races and branches of the state $\mathbf{C 3}$ are of the type $(0, \hat{\alpha})$ where the balls are split in one race and remain coincident in the other. For the ABB system with eccentricity $\epsilon$, the coefficients satisfy $a_{1}+a_{2}=M \epsilon / 8 R$ and the bifurcation type is confined to lie on the thick dashed line. If $\Omega_{\text {con }}>\Omega_{\text {cyl }}$ then as $\Omega$ increases the pitchfork type will follow the upper route passing from (ii)

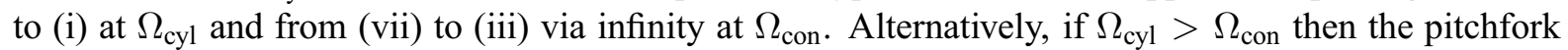
will follow the lower route initially switching from (iii) to (vii) at $\Omega_{\text {con }}$ and then passing from (i) to (ii) at $\Omega_{\text {cyl }}$. 
bifurcation type for the ABB system must always lie on the thick dashed line. The position of the pitchfork on this line is then determined by $\kappa(\Omega)$. For example, if we assume that the balancing planes are inside the bearings so that $z<l$, then by (32) we have $0<\kappa(0)<1$. Therefore, from the expressions for the coefficients (34), we have $a_{1}>a_{2}>0$, and so, the bifurcation type for $\Omega=0$ starts in region (ii).

With regards to Figure 7 (a), if $\Omega_{\text {con }}>\Omega_{\text {cyl }}$ then $k^{\prime}(\Omega)<0$. Therefore, from equation (34) we find that as $\Omega$ increases, $a_{1}$ decreases and $a_{2}$ increases. Thus, the solution type always moves upwards and to the left and follows the upper route. As $\Omega$ passes through $\Omega_{\text {cyl }}$ the pitchfork crosses from region (ii) to (i) and the solutions $\mathbf{B}$ and $\mathbf{C 3}$ exchange stability. Next, as $\Omega$ increases further, the bifurcation type goes from (i) to (vii) and C3 also loses its stability. Then as $\Omega$ passes through $\Omega_{\text {con }}, \kappa$ switches from $-\infty$ to $+\infty$ and the pitchfork traverses, via infinity, from region (vii) to (iii). Hence, the stability of the balanced state $\mathbf{B}$ is regained.

The situation is similar for the less common set-up with $\Omega_{\text {cyl }}>\Omega_{\text {con }}$. With regards to Figure $7(\mathrm{~b})$, we have that $k^{\prime}(\Omega)>0$, and so the solution type moves down and to the right as $\Omega$ increases. The balanced state B initially becomes unstable as $\Omega$ passes through $\Omega_{\text {con }}$ and the pitchfork switches via infinity from region (iii) to (vii). Its stability is later regained when the bifurcation type crosses from (i) to (ii) as $\Omega$ passes through $\Omega_{\text {cyl }}$.

The $\mathcal{D}_{4}$-equivariant bifurcation has now been characterised as it applies to a two-plane ABB. Furthermore, we have shown that the $\mathbf{B}$ type solution is stable at the pitchfork, provided that either $\Omega<\Omega_{1}$ or $\Omega>\Omega_{2}$. However, as mentioned above, the stability of the balanced state in the full system will be influenced by further bifurcations.

For example, when $\Omega<\Omega_{1}$, the trivial solution $\mathbf{C 1}$ of the pitchfork is unstable whereas the stable branch is the corresponding $\mathbf{C} \tilde{1}$ state with coincident balls on the heavy side of the race. This behaviour for subcritical speeds is well established [7, 13] and can be understood intuitively by considering the 'Working principle of the ABB', see [23, §4]. More recently, Green et al. [5] demonstrated that the solutions $\mathbf{C 1}$ and $\mathbf{C} \tilde{\mathbf{1}}$ are connected via a saddle-node bifurcation. It was also shown that the states swap stability as the saddle-node passes through the pitchfork at the codimension-two bifurcation that occurs at $(m, \Omega)=\left(m_{c}, \Omega_{1}\right)$.

For $\Omega>\Omega_{2}$ the stability of the balanced state $\mathbf{B}$ is determined by the Hopf bifurcation curves that denote the onset of oscillatory instabilities. These boundaries were discussed in $[5,6]$ and their computation will again form much of the basis for the remainder of this paper. First, let us return to a consideration of the symmetry of the imbalance, this time focusing on the unfolding of the $\mathcal{D}_{4}$ equivariant pitchfork as a misalignment is introduced. 


\subsection{Symmetry breaking between the races}

The up-down symmetry of the ABB is broken when there is more imbalance at one race than at the other. We are then left with the two ball interchange symmetries $\gamma_{12}=\left(\alpha_{1}, \dot{\alpha}_{1}\right) \leftrightarrow\left(\alpha_{2}, \dot{\alpha}_{2}\right)$ and $\gamma_{34}=\left(\alpha_{3}, \dot{\alpha}_{3}\right) \leftrightarrow\left(\alpha_{4}, \dot{\alpha}_{4}\right)$, and together these form the symmetry group of the rectangle $\mathcal{D}_{2}$. The appropriate reduced model can be adapted from the $\mathcal{D}_{4}$-equivariant normal form (20) by breaking the imbalance symmetry between the races, this gives

$$
\left(\begin{array}{l}
\mathrm{d} \hat{\alpha}_{12} / \mathrm{d} t \\
\mathrm{~d} \hat{\alpha}_{34} / \mathrm{d} t
\end{array}\right)=\left(\begin{array}{c}
\mu_{1} \hat{\alpha}_{12} \\
\mu_{2} \hat{\alpha}_{34}
\end{array}\right)-a_{1}\left(\begin{array}{c}
\hat{\alpha}_{12}^{3} \\
\hat{\alpha}_{34}^{3}
\end{array}\right)-a_{2}\left(\begin{array}{c}
\hat{\alpha}_{34}^{2} \hat{\alpha}_{12} \\
\hat{\alpha}_{12}^{2} \hat{\alpha}_{34}
\end{array}\right)
$$

with

$$
\mu_{1}=m-m_{t} \quad \text { and } \quad \mu_{2}=m-m_{b}
$$

Here $m_{t}$ and $m_{b}$ are the critical ball masses for the top and bottom races respectively.
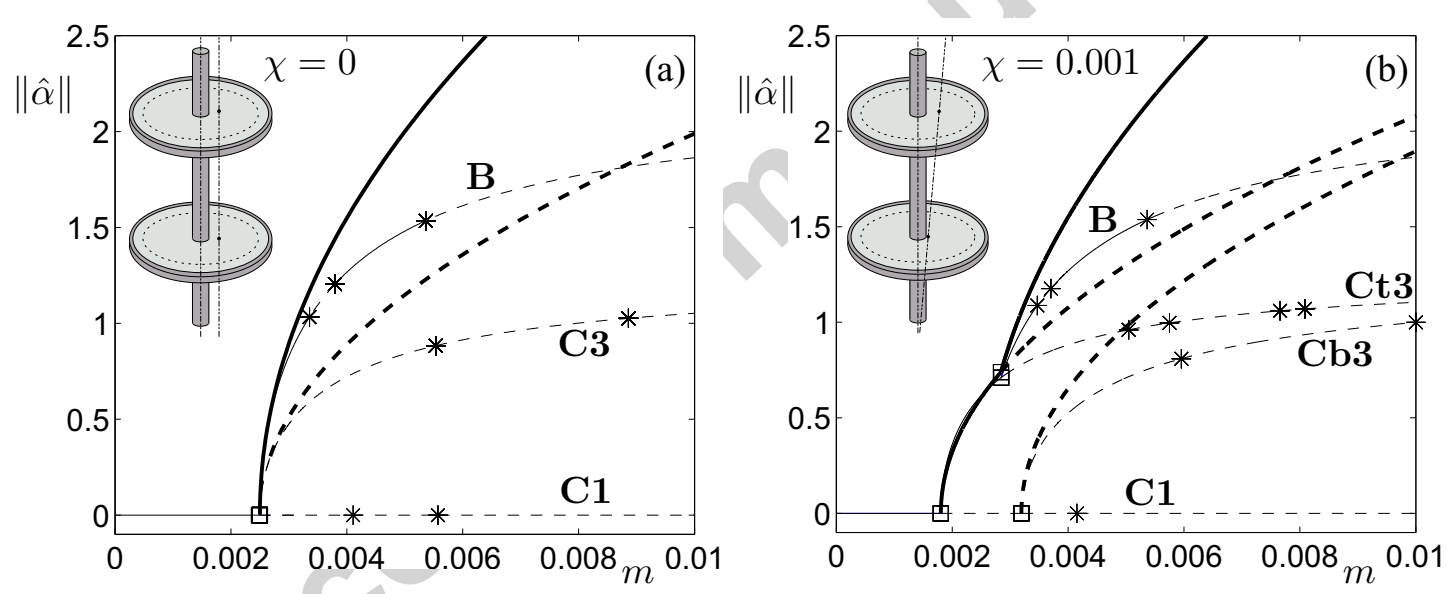

Figure 9: Comparison of the unfolding of the $\mathcal{D}_{4}$-equivariant pitchfork as a small misalignment $\chi$ is introduced. The parameter values are the same as that of Figure 5, however, the norm is now given by $\|\hat{\alpha}\|=\sqrt{\hat{\alpha}_{12}^{2}+\hat{\alpha}_{34}^{2}}$. The thin curves are the solutions to the full system (2) and (4), and the thick curves are the approximations that are computed from the reduced models (20) for (a) and (39) for (b).

The unfolding of the $\mathcal{D}_{4}$-equivariant pitchfork is illustrated in Figure 9. The thin curves are the solutions of the full system, and except for the measure which is now $\|\hat{\alpha}\|=\sqrt{\hat{\alpha}_{12}^{2}+\hat{\alpha}_{34}^{2}}$, these results are the same as those of Figure 5. The thick curves are the approximations that have been computed from the reduced models (20) for panel(a) and (39) for (b). We find that the unfolding takes the correct form and also that the relative stabilities of the solutions correspond. Furthermore, the quantitative match is good, and this is especially true for the $\mathcal{D}_{4}$ case (a). We note again that the method for finding the reduced model solutions was to calculate the best quadratic approximations to the branches of the 
$\mathcal{D}_{4}$-equivariant bifurcation in the full system. Also, one should not expect to find the secondary Hopf bifurcations as this is beyond the scope of the normal form.

The advantage of investigating symmetric configurations is that the solution structure of the resulting bifurcations is often affected or determined by the symmetry properties of the experimental set-up. Furthermore, it is relatively easy to analyse small deviations from the symmetric case by unfolding the bifurcation in the appropriate manner. A relevant extension to this work would be the characterisation of the codimension-two pitchfork-Hopf bifurcations that give rise to the Hopf instability curves. This should first be carried out for an isotropic single-plane ABB, before then proceeding onto more complicated mechanical systems. However, even for the simplest case, it is likely that the inclusion of important damping effects could make the problem intractable [2]. Therefore, we prefer to proceed with a numerical bifurcation analysis in which further asymmetries of a two-plane ABB are considered.

\section{Support asymmetry}

As discussed previously, rotating machines often run on bearings that have different stiffness and damping characteristics. This usually occurs due to an asymmetric geometry of the external support structure. The installation of a gas turbine below an aeroplane wing is one such example, and this configuration leads to bearings which have directionally dependent support stiffnesses. In this section we shall investigate how these effects influence the stability of the balanced state.

We consider the model given by (2-4), which is written with respect to inertial space frame coordinates, and includes the effect of support anisotropy. We again assume a constant rotation speed $\dot{\theta}_{0}=\Omega$, and so equation (3) will be neglected. The continuation package AUTO [21] is used to compute bifurcation diagrams that show the stable regions of the balanced state $\mathbf{B}$ in various parameter planes. Because equations (2) and (4) are periodically forced by the imbalance, the time $t$ only enters explicitly in the form of $\sin (\Omega t)$ and $\cos (\Omega t)$. This property enables the system to be rendered autonomous by appending the 2D non-linear oscillator $[21, \S 10.5]$

$$
\begin{aligned}
& \dot{s}=s+\Omega c-s\left(s^{2}+c^{2}\right), \\
& \dot{c}=-\Omega s+c-c\left(s^{2}+c^{2}\right),
\end{aligned}
$$

and then substituting its asymptotically stable solution $s=\sin (\Omega t)$ and $c=\cos (\Omega t)$ back into the other equations. Hence, the fixed points of the isotropic rotating frame system of Section 3 can be identified as circular periodic orbits in the present fixed frame system. Consequently, as the balanced state is now viewed as a limit cycle, the stability boundaries will be formed by torus bifurcations as opposed to Hopf bifurcations.

We also remind the reader that the stability results that will be presented are only valid locally and 
there most likely exists competing dynamics in much of the stable range. A Lyapunov function could in theory be constructed to determine where the dynamics are globally stable. However, this type of analysis lies outside the scope of the present work. Nevertheless, numerical continuation techniques have recently been successfully employed during the first stage of the design process for a practical automatic balancing mechanism. Numerical time series simulations were then carried out to determine the global stability of the device. Finally, a single-plane balancing test rig was built to validate some aspects of the model, see reference [27] for further details.

\subsection{Isotropic supports $-\mathbf{K}_{X}=\mathbf{K}_{Y}$ and $\mathbf{C}_{X}=\mathbf{C}_{Y}$}

\subsubsection{Uncoupled translational and inclinational support properties}

When the stiffness and damping matrices have no off-diagonal terms, the rotor's translational and inclinational degrees of freedom are only coupled through the motion of the balancing balls. This situation can occur, for instance, in the case of a rigid rotor on two equal bearings with the centre of mass exactly at the midspan. First, we consider the isotropic system with parameters given by (10) so that the stiffness and damping matrices are

$$
\mathbf{K}_{X}=\mathbf{K}_{Y}=\left[\begin{array}{ll}
1 & 0 \\
0 & 9
\end{array}\right] \quad \text { and } \quad \mathbf{C}_{X}=\mathbf{C}_{Y}=\left[\begin{array}{cc}
0.02 & 0 \\
0 & 0.18
\end{array}\right]
$$

Figure 10 (a) illustrates the stability diagram for the static imbalance case. The eccentricity $\epsilon / R$ is plotted against $\Omega$, whilst we also vary the ball mass so that $m / M=\epsilon / R$. Thus, the ball mass scales with the imbalance and so the balanced state ball positions do not change value. Physically the condition $m / M=\epsilon / R$ means that each ball has enough mass to compensate for the rotor eccentricity. Therefore, as there are a total of four balls, the $\mathrm{ABB}$ is at $25 \%$ of its balance correction limit. In addition, a logarithmic scale is used for the vertical axis so that a wide range of eccentricities can be considered. The main area of interest for applications occurs where there is a large connected stable region for small eccentricities and supercritical rotation speeds. The torus instability curve which bounds this region asymptotes towards $\Omega=\Omega_{\text {con }}$ as $\epsilon / R=m / M \rightarrow 0$, hence, there is no stable region in the subcritical regime.

A similar plot is illustrated in Figure 10 (b) for a dynamic imbalance, that is to say, an imbalance which has both an eccentricity $\epsilon \neq 0$ and a misalignment $\chi \neq 0$. Here, we take $m / M=\epsilon / R+\chi$, $\epsilon / R=\chi$ and constant phase $\beta=1$, and again the $\mathrm{ABB}$ is at $\approx 25 \%$ of its balance correction limit. We find that the regions of stability remain largely unchanged, however, extra torus curves are now present. As discussed in Section 3, these arise because the introduction of a small misalignment breaks the symmetry between the two races. 

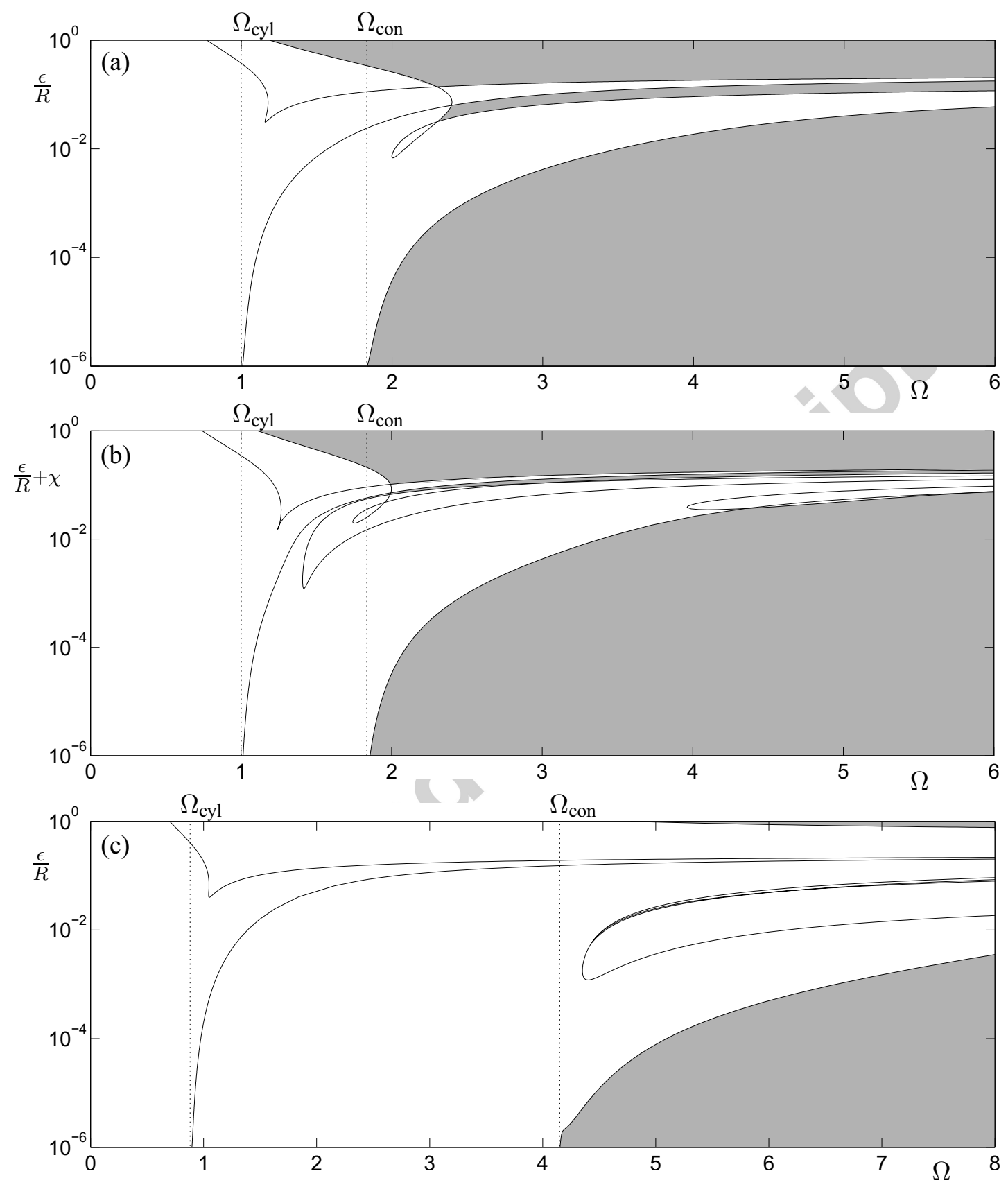

Figure 10: Stable regions of the balanced state (shaded) upon variation of $\epsilon / R$ against $\Omega$, whilst the ball mass is also varied so that the balanced state ball positions remain constant. Panels (a) and (b) are for a static and dynamic imbalance respectively, in both cases the translational and inclinational support properties are uncoupled, see (41). Panel (c) is for a static imbalance case in which the support parameters are given by equation (42). These values correspond to a coupled system in which the centre of mass $G$ is placed one quarter of the length along the shaft. 


\subsubsection{Coupled translational and inclinational support properties}

Next we consider the case in which the stiffness matrices remain isotropic, but where they also have off-diagonal terms. This would occur, for example, when the bearings have unequal stiffnesses or where the centre of mass is not at the midspan. Here, we take

$$
\mathbf{K}_{X}=\mathbf{K}_{Y}=\left[\begin{array}{cc}
1 & 3 \\
3 & 45
\end{array}\right] \text { and } \mathbf{C}_{X}=\mathbf{C}_{Y}=\left[\begin{array}{cc}
0.02 & 0.06 \\
0.06 & 0.90
\end{array}\right]
$$

which corresponds to a set-up with $l_{1}=9, l_{2}=3$ in which the rotor's centre of mass $G$ is three times further from one bearing than the other. This yields $\Omega_{\text {cyl }} \simeq 0.89$ and $\Omega_{\text {con }} \simeq 4.07$, for the critical speeds that are associated with the cylindrical and conical whirls respectively. Figure 10(c) shows the results for a static imbalance, and as in panels (a) and (b), bifurcation curves asymptote to the critical frequencies as the ball mass and eccentricity both tend to zero. The stable regions in the high eccentricity regimes have now almost disappeared, although more importantly, we find that the stable region for low eccentricities still exists, and has the same qualitative shape as before. We note that the results for the corresponding dynamic imbalance case are similar to those for the static imbalance case of Figure 10(c).

\subsection{Anisotropic supports}

Finally, we consider the case of anisotropic supports. The main feature of this system is the splitting of circular whirls into distinct elliptical orbits that have different resonant frequencies. In addition, the rotor may undergo a backward whirling response in between the split resonances, that is to say, the whirl orbit may rotate in the opposite direction to the rotor's spin. For further details, see for example [18, $\S 6],[24, \S 8.3]$ and $[25, \S 6.2 .5]$.

Here, we shall take the stiffness and damping matrices given by

$$
\mathbf{K}_{X}=\left[\begin{array}{ll}
1 & 0 \\
0 & 9
\end{array}\right], \quad \mathbf{K}_{Y}=\left[\begin{array}{cc}
5 & 0 \\
0 & 45
\end{array}\right] \quad \text { and } \quad \mathbf{C}_{X}=\left[\begin{array}{cc}
0.02 & 0 \\
0 & 0.18
\end{array}\right], \quad \mathbf{C}_{Y}=\left[\begin{array}{cc}
0.1 & 0 \\
0 & 0.9
\end{array}\right]
$$

in which the stiffness and damping in the $Y$-direction is 5 times greater than that in the $X$-direction. The approximate critical speeds have been numerically computed by determining the frequencies of the maximum response to imbalance. In this case, the translational and tilting critical frequencies are $\Omega_{\mathrm{cyl}, X}=1$ and $\Omega_{\mathrm{con}, X} \simeq 1.65$ in the $X$-direction, and are $\Omega_{\mathrm{cyl}, Y} \simeq 2.24$ and $\Omega_{\mathrm{con}, Y} \simeq 3.79$ for the $Y$-direction.

Figure 11 shows a stability chart for a static type imbalance c.f. Figure 10(a). The stable region for the high eccentricity regime no longer exists, however, new 'wedge' shaped stable regions now occur for low eccentricities when the speeds are in the vicinity of the rigid body resonances. The bifurcation 


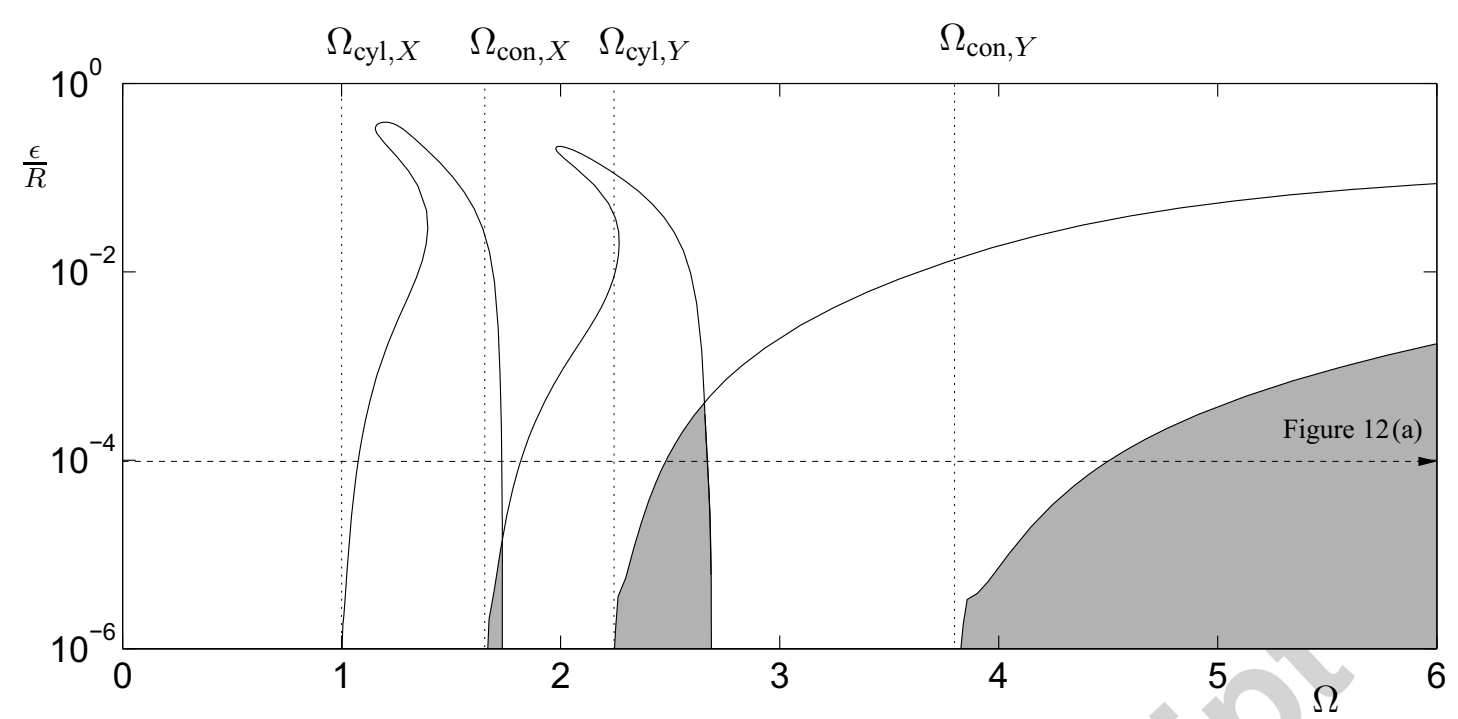

Figure 11: Stability chart for a set-up with anisotropic supports. Here the stiffness in the $Y$-direction is 5 times greater than that in the $X$-direction, see (43). A one parameter 'brute force' bifurcation diagram for the indicated section in $\Omega$ is displayed in Figure 12(a).

curves again emanate at the critical frequencies and this type of behaviour is reminiscent of a 'modelocking' phenomena in which coupled oscillators synchronize within specific parameter regions [26, $\S 3]$. The manner in which the stability regions of the ABB are influenced by the backward whirl orbits is a topic for future research. However, it is clear that there will be a complicated interdependence, and this relationship is also investigated for a particular single-plane ABB experimental set-up in [27].

Next, in Figure 12 we illustrate some 'brute force' bifurcation diagrams as $\Omega$ is varied, and $\epsilon / R=$ $m / M=1 \times 10^{-4}$ is held constant. The results were obtained as follows: for each value of $\Omega$ we let the transients die away, and for the long term solution we plot $\bar{A}$ which is the maximum value of the average rotor vibration at points one unit length from the midspan. The initial conditions were such that the balls started at rest with respect to the rotor and on opposite sides of the race, that is to say, they do not add to the initial imbalance. Therefore, physically these results correspond to a set of experiments in which the balls are clamped during the run-up, and then released when a constant operating speed is reached.

Panel (a) is for a static imbalance and illustrates the results for the indicated one-parameter section through Figure 11. At certain intervals between resonances and for frequencies in excess of the highest critical speed the ABB effectively eliminates rotor vibrations whereas for the plain rotor $\bar{A} \rightarrow \epsilon / R=$ $1 \times 10^{-4}$ as $\Omega \rightarrow \infty$. By contrast, the ABB performs far worse than the plain rotor when in the vicinity of the critical speeds. We note that as $\chi=0$ the conical resonances are not excited in the plain rotor, however, the balls still become unstable with respect to these modes. Also, the ABB seems to balance the rotor for a greater range of speeds than is predicted by the section through Figure 11. This discrepancy occurs because the symmetry of the initial conditions prevent the balls from destabilising as expected. 

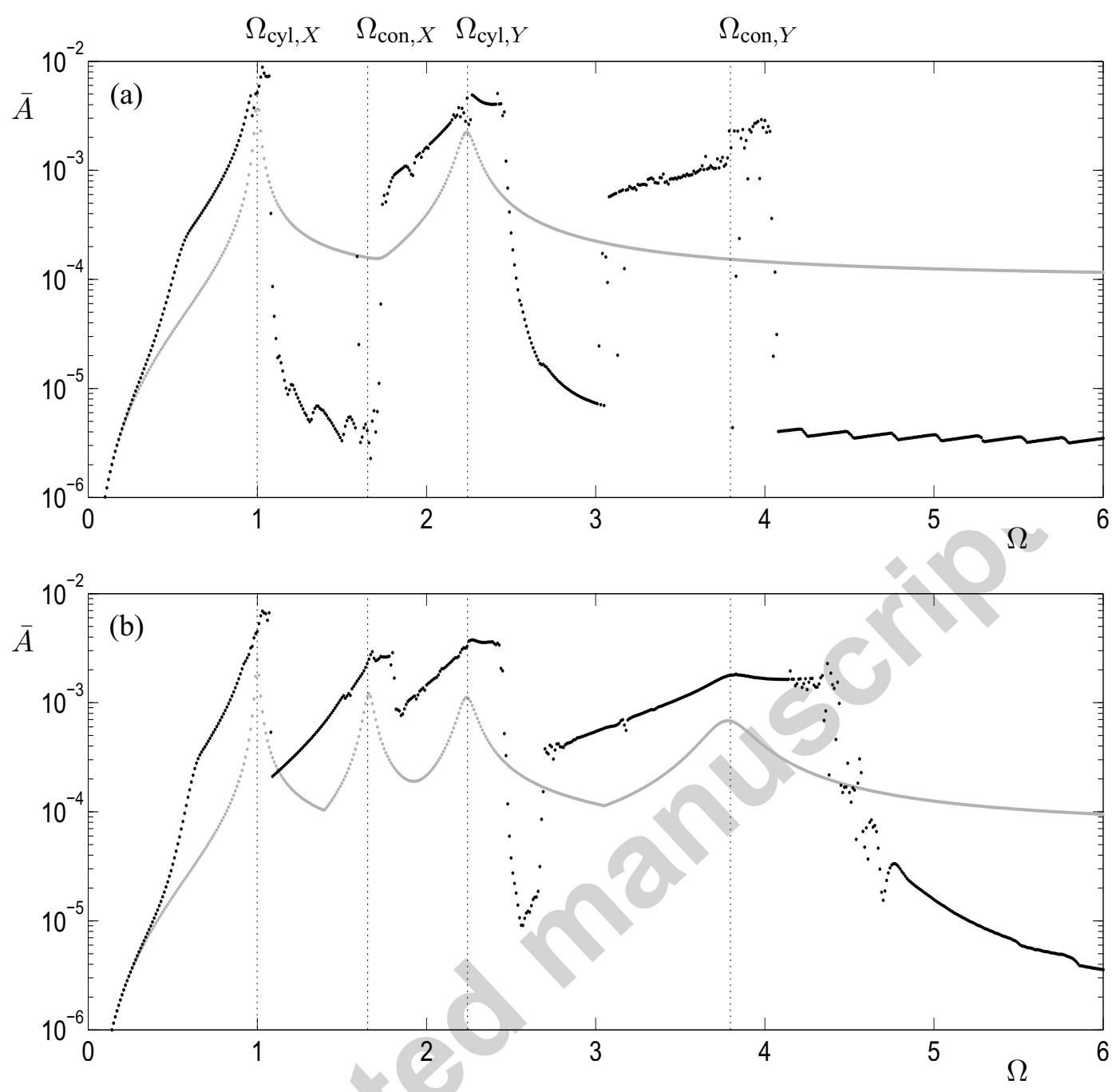

Figure 12: 'Brute force' bifurcation diagrams for a set-up with anisotropic supports. The rotor vibration $\bar{A}$ is plotted upon variation of $\Omega$ and the grey and black curves are for the plain rotor and ABB respectively. Panel (a) is for a static imbalance and illustrates the indicated one-parameter section through Figure 11; panel (b) is for a dynamic imbalance of a similar size.

Therefore, we also present results for the case of a dynamic imbalance with $m / M=\epsilon / R+\chi, \epsilon / R=\chi$ and a constant phase $\beta=1$. Here, there is a better agreement with the stability results of Figure 11 , and we find that the $\mathrm{ABB}$ still compensates for the imbalance in the highly supercritical frequency range.

\section{Device asymmetry}

A real ABB device will always possess unavoidable errors that arise during the manufacturing process. For example, the race centre can never be made to coincide exactly with the rotation centre and the resulting error is called the runway (or race) eccentricity. The influence of imperfections in the race geometry was investigated in detail by Huang et al. [4] and Olsson [28]. Here it was shown that the 
vibrational amplitude of an ABB is bounded below by its runway eccentricity, therefore it is important that this error is minimised.

In this section we shall consider other such ABB asymmetries. In a practical two-plane balancing procedure, design constraints often dictate that the balancing planes are not chosen to be equally spaced from the midspan, therefore, we will investigate the case in which $z_{1,2} \neq z_{3,4}$. In addition we shall consider a set-up where one ball is heavier than the others.

For ease of analysis, we will restrict attention to the isotropic case with

$$
\mathbf{K}_{X}=\mathbf{K}_{Y}=\mathbf{K}=\left[\begin{array}{ll}
1 & 0 \\
0 & 9
\end{array}\right] \text { and } \quad \mathbf{C}_{X}=\mathbf{C}_{Y}=\mathbf{C}=\left[\begin{array}{cc}
0.02 & 0 \\
0 & 0.18
\end{array}\right]
$$

Thus, we can use the autonomous rotating coordinates model that is given by (7) and (8). Also, unless otherwise stated, the values of the other parameters will again be given by (10). When the moments of inertia are such that $J_{t}>J_{p}$, the rotor is termed 'long' and there exists a critical speed $\Omega_{\text {con }}$ that is associated with the conical whirl. For the present case we have $J_{t}=3.25, J_{p}=0.5$ and so $\Omega_{\text {con }} \simeq 1.81$, see (11); these values correspond to a solid cylindrical rotor with a length of six times its radius.

Figure 13 shows various stability diagrams for a static type imbalance. As in Figure 10, the eccentricity $\epsilon / R$ is plotted against $\Omega$, whilst we also scale the ball mass so that the balanced state ball positions do not change value. Panel(a) serves as the control case and is the same (except for the aspect ratio) as that of Figure 10 (a). By contrast, the results for a 'disk' type rotor in which $J_{t}<J_{p}$ are shown in panel (b). Here, the influence of the gyroscopic terms are such that the eigenfrequency corresponding to the conical whirl is always greater than the rotor speed $\Omega$. This means that there is no conical critical speed $\Omega_{\text {con }}$ and thus no associated self-aligning process $^{2}$; hence the ABB is not stabilised with respect to conical motions. The method of direct separation of motion has been used by Sperling et al. [14] to derive this result and in addition they discuss how it relates to Blekhman's generalised self-balancing principle $[20, \S 8]$. From a practical viewpoint however, the prognosis for the autobalancing of 'disk' type rotors is not as bad as it may first seem. Because the conical mode has no associated critical speed, 'disk' rotors often need only to be balanced with respect to the static imbalance and a single plane ABB can be used to provide a partial imbalance compensation [29].

Next, we return to the 'long' type rotor case and investigate how the asymmetries of the ABB device can effect its stability. Panel (c) shows the situation where one of the balls has a mass that is $20 \%$ greater than the other balls. We see that the stable regions remain largely unchanged, however extra Hopf instability curves are present. These arise because the introduction of a different ball mass breaks the symmetry of the system. Another factor which must be noted is that balls of different mass cannot counterbalance each other by settling to opposite sides of the race. Thus the overall capability of the

\footnotetext{
${ }^{2}$ Self-aligning is the phenomena whereby a rotor will tend to rotate about its principal axis of inertia at supercritical rotation speeds.
} 

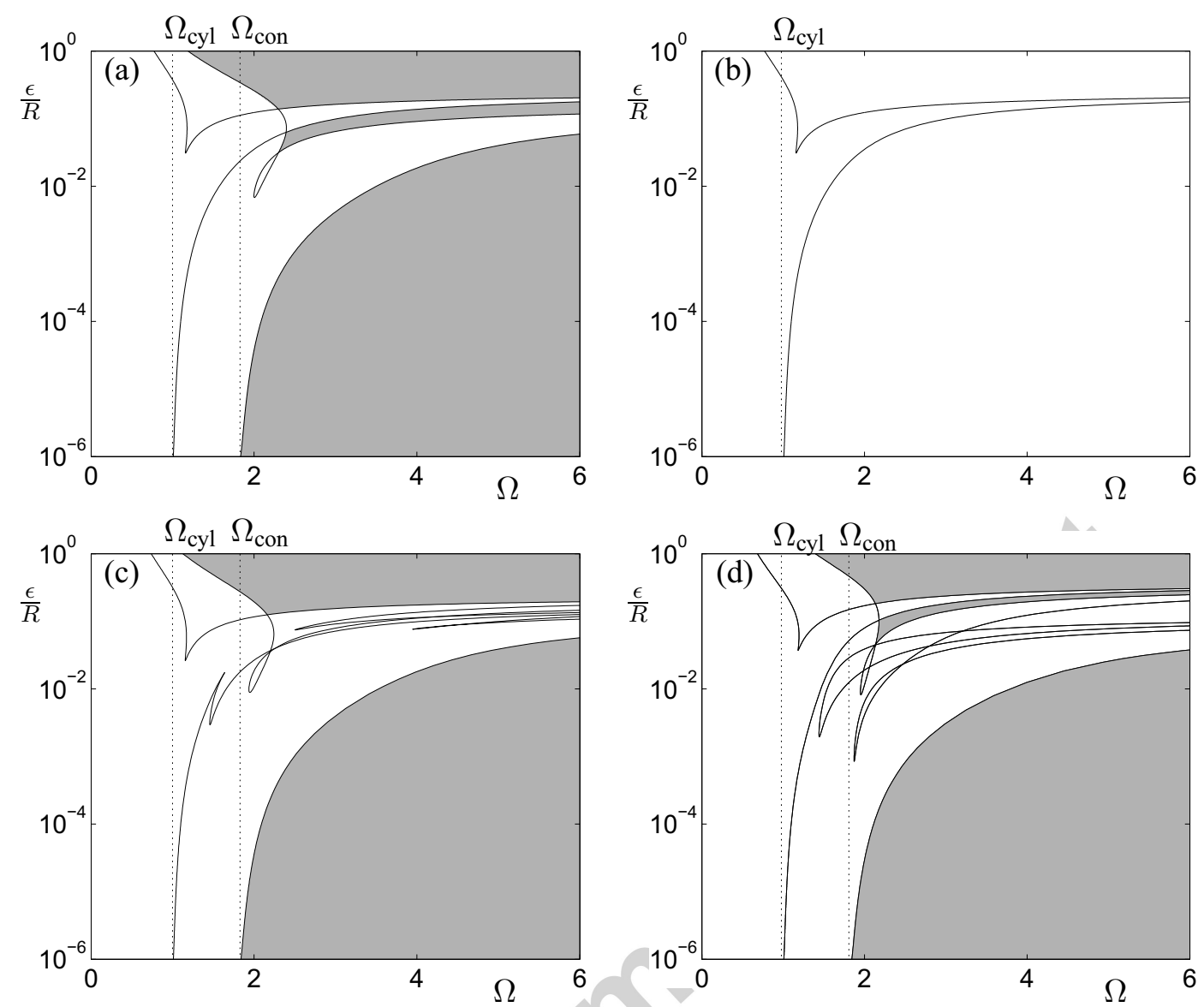

Figure 13: Bifurcation diagrams showing stable regions of the balanced state (shaded) in the case of a static imbalance. The eccentricity $\epsilon / R$ is varied against $\Omega$, whilst $m / M$ is kept equal to $\epsilon / R$ so that the balanced state ball positions remain constant. Panel (a) is a bifurcation diagram for a 'long' type rotor $J_{p}>J_{t}$ and panel (b) is for a 'disk' type rotor $J_{p}<J_{t}$. Similar diagrams for the 'long' type rotor are shown in (c) where one of the balls has a mass $20 \%$ greater than the others and in (d) where $z_{1,2}=1$ and $z_{3,4}=3$ so that the balancing planes are not equidistant from the midspan.

$\mathrm{ABB}$ is reduced as unequal balls will inevitably add an imbalance to a rotor that is already well balanced [30]. Next the diagram in panel (d) shows the case where the balancing planes are not equally spaced from the midspan. We have taken $z_{1,2}=1$ and $z_{3,4}=3$, and again the main point to note is the robustness of the stable region for low eccentricities and supercritical rotation speeds.

We shall now return to the symmetric $\mathrm{ABB}$ set-up and consider a rotor that suffers from a dynamic imbalance. Similar plots to those of Figure 13 are illustrated in Figure 14 for the dynamic imbalance case with $m / M=\epsilon / R+\chi, \epsilon / R=\chi$ and a constant phase $\beta=1$. In panel (a) the race damping parameter is $\bar{c}_{b}=0.01$, whereas for panel (b) the race damping value has been increased to $\bar{c}_{b}=0.1$. As a consequence much of the complicated structure in the high eccentricity regime has been smoothed out.

The limit cycle which is born at the marked Hopf bifurcation for $\bar{c}_{b}=0.01$ and $\epsilon / R+\chi=0.01$ 

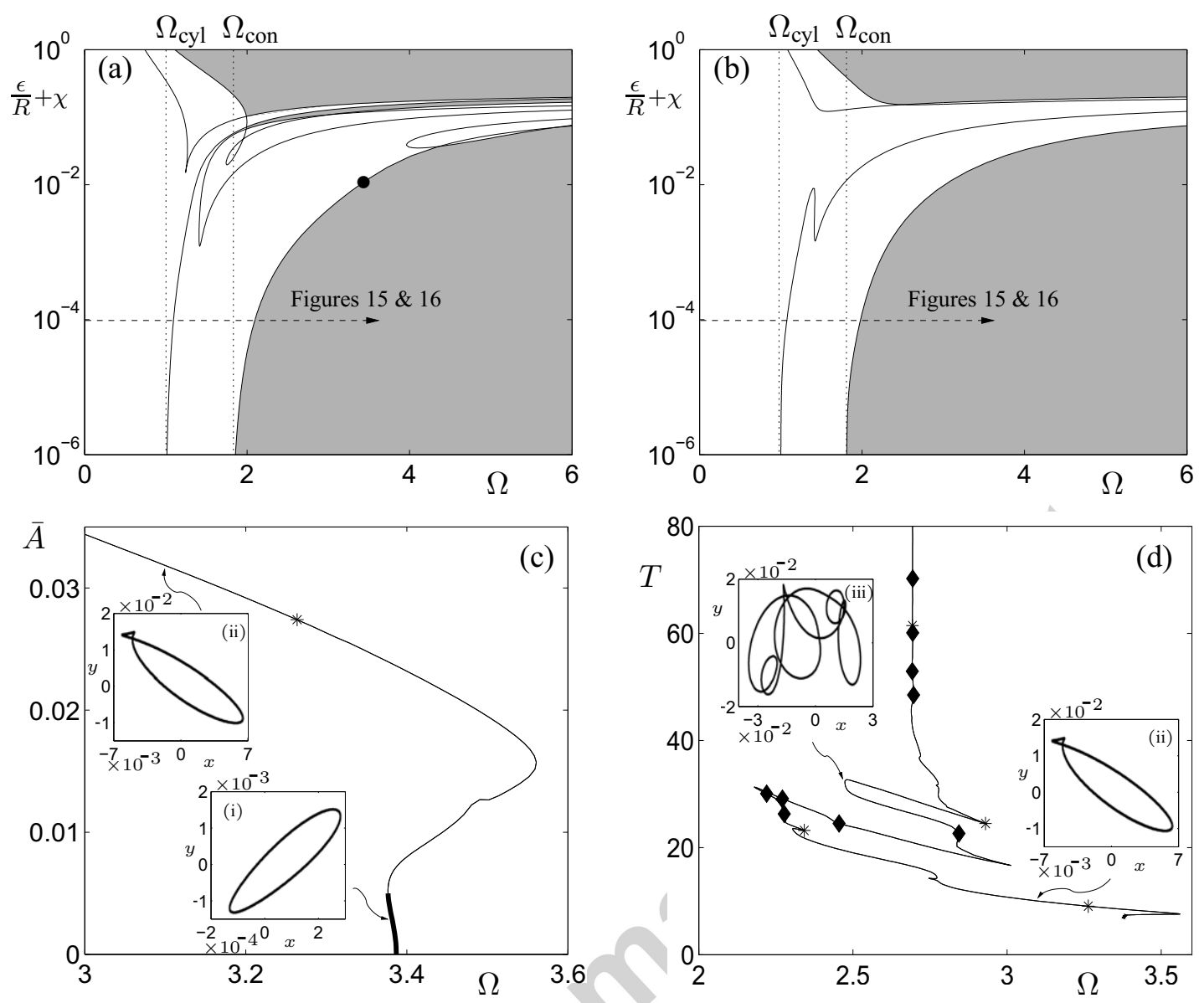

Figure 14: Bifurcation diagrams in the case of a 'long' rotor with a dynamic imbalance. Panel (a) is for a race damping value of $\bar{c}_{b}=0.01$ and panel (b) is for a higher race damping value of $\bar{c}_{b}=0.1$. The indicated one-parameter sweeps in $\Omega$ are illustrated in Figures 15 and 16. Panel (c) illustrates a continuation of the periodic orbit that emanates from the Hopf bifurcation marked by a (•) in (a). Here, $\bar{A}$ is the vibration norm and the thick and thin curves represent stable and unstable limit cycles respectively. In panel (d) this branch is continued further in order to show its long-term behaviour, here $T$ is the period of the orbit. Torus bifurcations are denoted by a $(*)$ and period-doubling bifurcations by a $(\checkmark)$. The insets illustrate the whirl orbits in the (rotating) $x$-y plane of the geometric centre $C$, at (i) $(\Omega, T)=(3.386,7.081$ ) (stable), (ii) $(\Omega, T)=(3.127,9.856)$ (unstable) and (iii) $(\Omega, T)=(2.475,32.453)$ (unstable).

is continued in panel (c), here the measure $\bar{A}$ is the average rotor vibration at points one unit length from the midspan. We find that the Hopf bifurcation is supercritical and so there is a small region, as indicated by the bold curve, in which the limit cycle is stable. Therefore, in a controlled experiment we would expect, as $\Omega$ is decreased through this bifurcation, to see small oscillations of the balls about the balanced positions. The balls would then desynchronize with the rotor if $\Omega$ was reduced still further. For smaller values of the imbalance, say $\epsilon / R+\chi=1 \times 10^{-4}$, we have found that the Hopf bifurcation is subcritical and the transition to the desynchronized state would be immediate. The ability to follow the desynchronized limit cycles with continuation software is a topic for future work. Finally, panel (d) illustrates the long-term behaviour of the branch of limit cycles that was plotted in (c). We find that the 
solution 'cuts-back' on itself so that the period of the orbit does not increase monotonically. In addition, the insets show that the successive whirl orbits have an increasing number of loops. This behaviour, which was first described for a single plane ABB by Green et al. [5], is similar to the zipper bifurcation mechanism in which mode-locking periodic orbits merge with a homoclinic bifurcation at a resonance [31].

\section{Effect of the rotor run-up}

As yet, we have only considered systems in which the rotor speed $\Omega$ is assumed constant. However, if an $\mathrm{ABB}$ is to reach a stable region for balanced operation, then it must necessarily pass through at least one critical speed. Furthermore, the ABB usually increases the vibration levels of the rotor in the vicinity of a critical speed. In order to resolve this problem, various designs have been put forward in which the balls are locked in place during the rotor's acceleration phase. For example, Thearle's original 1932 ABB invention incorporated a hand operated clutch [7], and a constraint system was also utilised by Horvath et al. for a pendulum balancer [30]. Nevertheless, clamping mechanisms often detract from the simplicity of the design and can also fail to release the balls at the desired speeds. Thus, the majority of commercial ABBs do not have any locking mechanism for the balls.

This motivates a consideration of the ABB dynamics during the rotor run-up. In order to simplify the system, we will restrict attention to the isotropic case which has an imposed spin speed $\Omega=\Omega(t)$; the model for this set-up is given by (2) and (4).
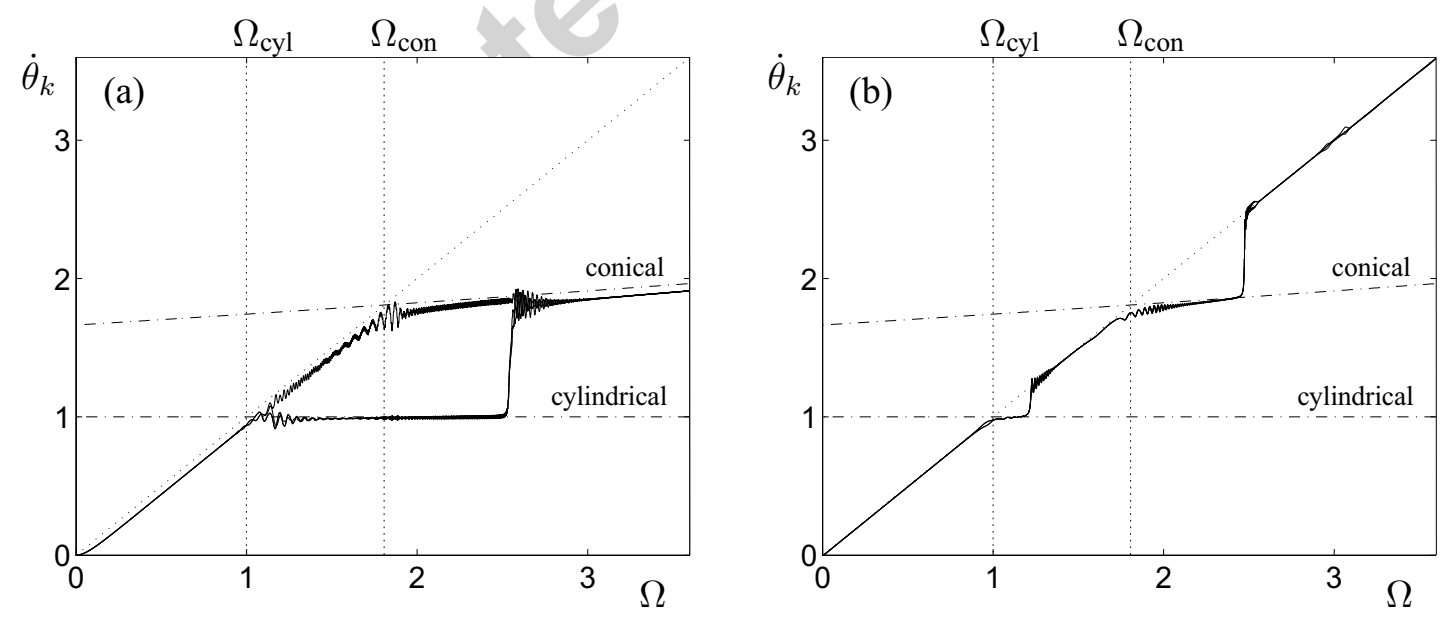

Figure 15: Diagram showing the ball speeds $\dot{\theta}_{k}, k=1 \ldots 4$ against the rotor speed $\Omega$ for the dynamic imbalance case with $\epsilon / R+\chi=1 \times 10^{-4}$. Panel (a) is for a race damping value of $\bar{c}_{b}=0.01$ and panel (b) is for a value of $\bar{c}_{b}=0.1$, see Figures 14(a) and (b) for the corresponding sweeps in parameter space. The rotation speed increases from $\Omega=0$ to $\Omega=3.6$ with a constant acceleration over a time scale of $\Omega_{\text {cyl }} t=6 \times 10^{3}$. Initial conditions are such that the rotor starts at rest in the undeflected position with the balls on opposite sides of the race. 

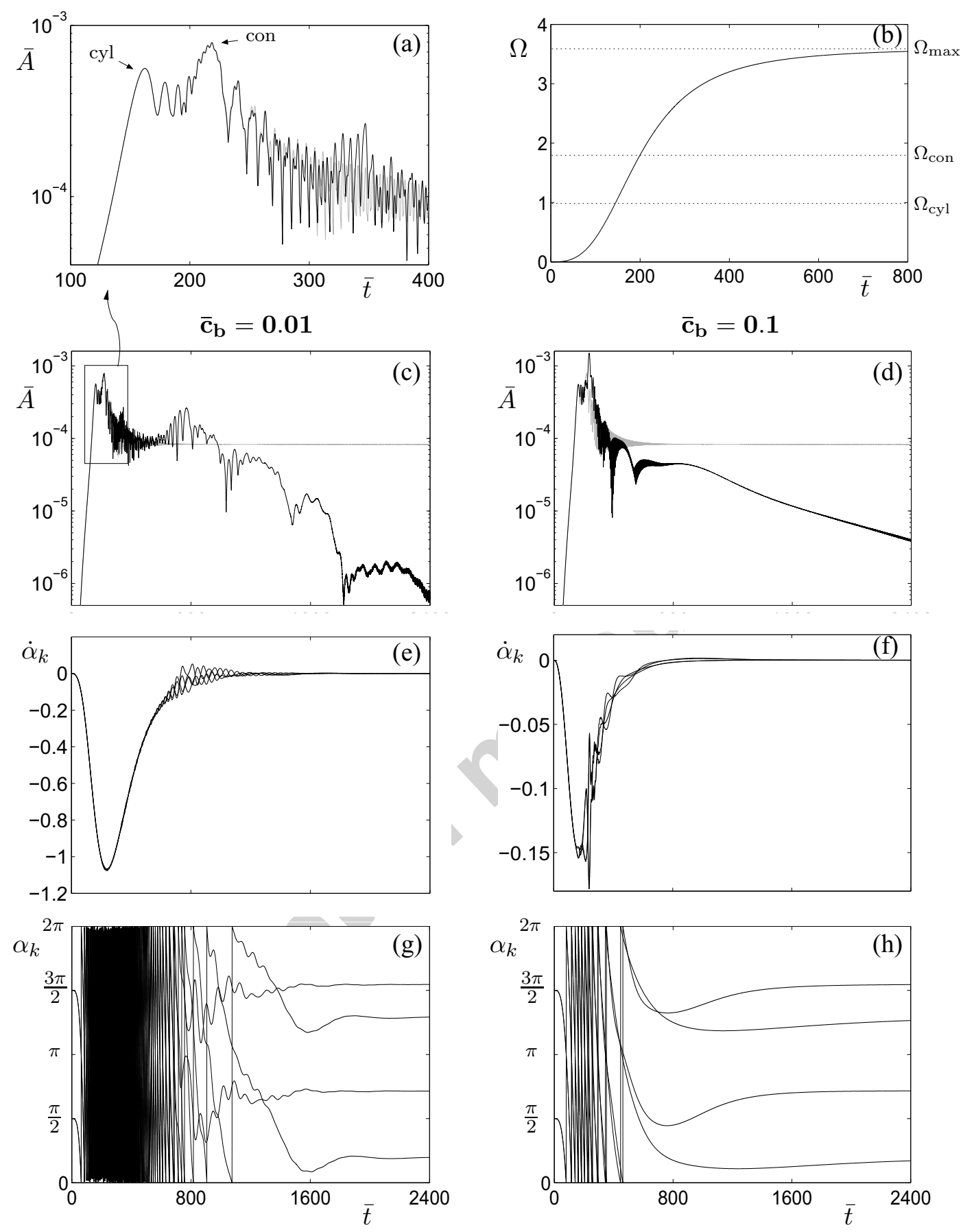

Figure 16: Simulations which include the effect of rotor run-up, the system parameters are the same as that for sweeps of Figure 15. Panel (b) shows the considered angular velocity profile. The case with $\bar{c}_{b}=0.01$ is displayed on the left column. Panel (a) shows the indicated detail of (c) in which the vibration levels $\bar{A}$ for the ABB (black curve) and the plain rotor (grey curve) are plotted. Panel (e) illustrates the ball speeds $\dot{\alpha}_{k}$ relative to the race and panel (g) displays the ball phases $\alpha_{k}$. The corresponding plots for a higher race damping value of $\bar{c}_{b}=0.1$ are given in panels (d), (f) and (h). In both cases the initial conditions are again such that the rotor starts at rest in the undeflected position with the balls on opposite sides of the race. 
In Figure 15 we plot the absolute ball speeds $\dot{\theta}_{k}$ against the rotor speed $\Omega$ for the sweeps with $\epsilon / R+\chi=1 \times 10^{-4}$ that are shown in the dynamic imbalance stability charts of Figures 14 (a) and (b). We slowly and uniformly increase the rotation speed over a time scale of $\Omega_{\text {cyl }} t=6 \times 10^{3}$. For a rotor with $\Omega_{\mathrm{cyl}}=1000 \mathrm{rpm}$, this corresponds to a constant acceleration phase that lasts approximately 1 minute with a final operating speed of $\Omega=3600 \mathrm{rpm}$.

Panel (a) illustrates the case for $\bar{c}_{b}=0.01$, here the race damping value is too low and the $\dot{\theta}_{k}$ curves lie below the line $\dot{\theta}=\Omega$. Thus we can infer that the balls lag the rotor and whirl backwards with respect to the race. Furthermore, as the rotor approaches and passes through its critical speeds, the balls tend to 'stall' and synchronize at a speed just below the rotor eigenfrequencies. The resulting vibration levels are far higher than that of the rotor without the ABB. Therefore, this example serves to illustrate that even if the balanced state is locally stable, the conditions during the run-up can prevent the ABB from achieving balance. The stalling behaviour, which was first analysed for a single plane ABB by Ryzhik et al. [32], is similar to the Sommerfeld effect in which a rotating machine with an insufficiently powerful motor has difficulty in passing through the critical speeds.

Figure 15(b) is a plot of the corresponding results for the higher race damping value of $\bar{c}_{b}=0.1$. We find that the $\dot{\theta}_{k}$ again stall as the rotor passes through the critical speeds, however, as $\Omega$ is increased still further the balls resynchronize with the rotor and eventually this leads to balanced operation.

The optimisation of the velocity profile for a particular application lies outside the scope of the present study, however, we shall now consider a more realistic rotor run-up that we can model by the Hill function

$$
\Omega(\bar{t})=\Omega_{\max } \frac{\bar{t}^{n}}{\bar{t}_{1 / 2}^{n}+\bar{t}^{n}} \quad \text { where } \bar{t}=\Omega_{\text {cyl }} t
$$

This velocity profile is plotted in Figure 16(b) with the parameter values $\left(\Omega_{\max }, \bar{t}_{1 / 2}, n\right)=(3.6,200,3)$. For a set-up with $\Omega_{\mathrm{cyl}}=1000 \mathrm{rpm}$, this profile would correspond to the rotor passing through the critical speeds during the 1 to 2 second time interval. We use this run-up to perform the same sweeps as for Figure 15, and the initial conditions are again such that the balls start on opposite sides of the race.

Figures 16 (a), (c), (e) and (g) illustrate the case for low race damping $\bar{c}_{b}=0.01$. Panel (a) shows a zoom of the run-up transient and we find that the rotor passes through its critical speeds by $\bar{t}=250$. At this point the ball speeds lag behind $\Omega$, however, they eventually catch up and begin to synchronize with the rotation speed, panel (e). Furthermore, they have phases which compensate for the rotor imbalance, that is to say, the ABB eventually achieves balanced operation, see panels (c) and (g). It is interesting that there is a period of increased vibrations as the balls approach the rotor speed. This occurs because the balls move from their positions on opposite sides of the race and begin to oscillate before they eventually synchronize with the rotor. 
The resulting vibrations at around $\bar{t} \approx 800$ can be reduced if the race damping parameter is increased to say $\bar{c}_{b}=0.1$, see panels (d), (f) and (h). In this case the balls take less time to reach the speed of the rotor. However, during the rigid body resonance regime, the balls add to the imbalance and the vibration levels at the critical speeds are higher than that of the plain rotor. Nevertheless, and as mentioned previously, the lagging motions of the balls can be eliminated by using clamping mechanisms [7] or partitioned races [11].

\section{Conclusion}

In this paper, we have presented a simple model for a two-plane automatic ball balancer (ABB). The use of complex coordinates enabled the equations to be written in a compact form, and effects such as support anisotropy and rotor acceleration were also included. We have shown that for a symmetric set-up the balanced state is born at a $\mathcal{D}_{4}$-equivariant pitchfork bifurcation. The solution structure of the relevant normal form was characterised in relation to the ABB system parameters and the unfolding of the symmetric bifurcation was described as a different amount of imbalance was introduced to each race. Furthermore, the symmetry properties of this system have enabled us to demonstrate that operation above both critical frequencies is a necessary but not a sufficient condition for the stability of the balanced state.

Next, two-parameter bifurcation diagrams were obtained through the numerical continuation of Hopf and torus instability curves. We show that, if the machine has a small imbalance and is operating above the rigid body resonances, then the addition of support and device asymmetries have little effect on the stable region of the balanced state. For example, in gas turbine and machine tool applications, typical eccentricities are $\epsilon / R \approx 1 \times 10^{-5}$ [18, Appx. B]; and we find that with these values, the balanced state is stable for speeds just above the highest critical speed. However, for washing machine applications the imbalances are around $\epsilon / R \approx 1 \times 10^{-2}$ [3], and the $\mathrm{ABB}$ remains unstable at frequencies that are far in excess of the critical speeds.

Finally, we considered the influence of the rotor run-up. Here, it was demonstrated that if the value of the race damping is too small, then the balls can 'stall' as the rotor passes through a critical speed. In addition, we have found that if the balls initially lag behind the rotor during a rapid run-up, then an increase in vibrations can occur as the balls desynchronize with each other before they reach the balanced state.

Even though the prospects for incorporating ABBs into high precision rotating machines seem promising, the stringent tolerances that are required for such applications present further difficulties with regards to implementation. For example, as the considered eccentricity range becomes smaller, the impact of ball positioning errors due to geometric defects and race friction become ever more important. Resonances due to shaft flexibility may also be present in the supercritical frequency range and these 
responses can lead to a destabilisation of the balancing balls [33]. Therefore, for practical applications it is important to compute the critical speeds of the flexible modes using, for example, a finite element method rotordynamics package.

In order to assess the significance of such effects, experimental studies of specific ABBs are required and these have already been carried out for isotropic single-plane devices [9, 10, 23, 34]. In addition, a recent companion paper [27] investigates the dynamics of an experimental single-plane ABB system as it passes through multiple resonances. However, future work is still required in order to empirically test the performance of a two-plane ABB device.

\section{Acknowledgements}

DJR gratefully acknowledges the support from a CASE award provided by the EPSRC and Rolls-Royce plc.

\section{References}

[1] J. Lee and W. K. Van Moorhem. Analytical and experimental analysis of a self-compensating dynamic balancer in a rotating mechanism. Journal of Dynamic Systems, Measurement, and Control, 118(3):468-475, 1996.

[2] C. Rajalingham and S. Rakheja. Whirl suppression in hand-held power tool rotors using guided rolling balancers. Journal of Sound and Vibration, 217(3):453-466, 1998.

[3] S. Bae, J. M. Lee, Y. J. Kang, J. S. Kang, and J. R. Yun. Dynamic analysis of an automatic washing machine with a hydraulic balancer. Journal of Sound and Vibration, 257(1):3-18, 2002.

[4] W.-Y. Huang, C.-P. Chao, J.-R. Kang, and C.-K. Sung. The application of ball-type balancers for radial vibration reduction of high-speed optic disk drives. Journal of Sound and Vibration, 250(3):415-430, 2002.

[5] K. Green, A. R. Champneys, and N. J. Lieven. Bifurcation analysis of an automatic dynamic balancing mechanism for eccentric rotors. Journal of Sound and Vibration, 291(3-5):861-881, 2006.

[6] D.J. Rodrigues, A.R. Champneys, M.I. Friswell, and R.E. Wilson. Automatic two-plane balancing for rigid rotors. International Journal of Non-Linear Mechanics, 43(6):527-541, 2008.

[7] E. Thearle. A new type of dynamic-balancing machine. Transactions of the ASME, 54(APM-5412):131-141, 1932. 
[8] J. Chung and I. Jang. Dynamic response and stability analysis of an automatic ball balancer for a flexible rotor. Journal of Sound and Vibration, 259(1):31-43, 2003.

[9] Q. Yang, E.-H. Ong, J. Sun, G. Guo, and S.-P. Lim. Study on the influence of friction in an automatic ball balancing system. Journal of Sound and Vibration, 285(1-2):73-99, 2005.

[10] C.-P. Chao, C.-K. Sung, S.-T. Wu, and J.-S. Huang. Nonplanar modeling and experimental validation of a spindle-disk system equipped with an automatic balancer system in optical disk drives. Microsystem Technologies, 13(8-10):1227-1239, 2007.

[11] K. Green, A. R. Champneys, M. I. Friswell, and A. M. Muñoz. Investigation of a multi-ball, automatic dynamic balancing mechanism for eccentric rotors. Philosophical Transactions of the Royal Society A: Mathematical, Physical and Engineering Sciences, 366(1866):705-728, 2008.

[12] C.-J. Lu, M.-C. Wang, and S.-H. Huang. Analytical study of the stability of a two-ball automatic balancer. Mechanical Systems and Signal Processing, 23(3):884-896, 2009.

[13] M. Hedaya and R. Sharp. An analysis of a new type of automatic balancer. Journal of Mechanical Engineering Science, 19(5):221-226, 1977.

[14] L. Sperling, B. Ryzhik, Ch. Linz, and H. Duckstein. Simulation of two-plane automatic balancing of a rigid rotor. Math. Comput. Simul., 58(4-6):351-365, 2002.

[15] M. Golubitsky, I. Stewart, and D. G. Schaeffer. Singularities and Groups In Bifurcation Theory, Vol. 2. Springer-Verlag New York, LLC, 1988.

[16] R. Hoyle. Pattern Formation: An Introduction to Methods. CUP, 2006.

[17] C. P. Chao, C. T. Lee, and S. W. Shaw. Non-unison dynamics of multiple centrifugal pendulum vibration absorbers. Journal of Sound and Vibration, 204(5):769 - 794, 1997.

[18] G. Genta. Dynamics of Rotating Systems. Springer, 2005.

[19] L. Sperling, F. Merten, and H. Duckstein. Self-synchronization and automatic balancing in rotor dynamics. International Journal of Rotating Machinery, 6:275-285, 2000.

[20] I. Blekhman. Vibrational Mechanics. World Scientific, 2000.

[21] E. Doedel, A. Champneys, T. Fairgrieve, Y. Kusnetsov, B. Sanstede, and X. Wang. AUTO97: Continuation and bifurcation software for ordinary differential equations, 1997.

[22] P. Chossat and R. Lauterbach. Methods in Equivariant Bifurcations and Dynamical Systems. World Scientific, 2000. 
[23] N. van de Wouw, M. N. van den Heuvel, H. Nijmeijer, and J. A. van Rooij. Performance of an automatic ball balancer with dry friction. International Journal of Bifurcation and Chaos, 20(1):65-85, 2005.

[24] T. Yamamoto and Y. Ishida. Linear and Nonlinear Rotordynamics: A Modern Treatment with Applications. Wiley, 2001.

[25] M.I. Friswell, J.E.T. Penny, S.D. Garvey, and A.W. Lees. Dynamics of Rotating Machines. CUP, 2010.

[26] A. Pikovsky, M. Rosenblum, and J. Kurths. Synchronization: A Universal Concept in Nonlinear Sciences. CUP, 2001.

[27] D.J. Rodrigues, A.R. Champneys, M.I. Friswell, and R.E. Wilson. Experimental investigation of a single-plane automatic balancing mechanism for a rigid rotor. Preprint submitted to Elsevier, http: //hdl . handle. net/1983/1588, 2010.

[28] K.-O. Olsson. Limits for the use of auto-balancing. International Journal of Rotating Machinery, 10(3):221-226, 2004.

[29] L. Sperling, B. Ryzhik, and H. Duckstein. Single-plane auto-balancing of rigid rotors. Technische Mechanik, 24:1-24, 2004.

[30] R. Horvath, G. T. Flowers, and J. Fausz. Influence of nonidealities on the performance of a self balancing rotor system. Proceedings of IMECE, 2005.

[31] G. H M. van der Heijden. Bifurcation sequences in the interaction of resonances in a model deriving from nonlinear rotordynamics: the zipper. Dynamics and Stability of Systems, 15:159183(25), 2000.

[32] B. Ryzhik, L. Sperling, and H. Duckstein. Non-synchronous motions near critical speeds in a single-plane auto-balancing device. Technische Mechanik, 24:25-36, 2004.

[33] J. Ehyaei and M.M. Moghaddam. Dynamic response and stability analysis of an unbalanced flexible rotating shaft equipped with $n$ automatic ball-balancers. Journal of Sound and Vibration, 321:554-571, 2009.

[34] C.-P. Chao, C.-K. Sung, and H.-C. Leu. Effects of rolling friction of the balancing balls on the automatic ball balancer for optical disk drives. Journal of Tribology, 127(4):845-856, 2005. 
- Analysis of a two-plane automatic balancing device for rotating machinery.

- Support anisotropy, rotor acceleration and nonsymmetric imbalance considered in full.

- Mathematical bifurcation theory, backed up by numerical simulation results.

- The balanced state is found to be robust to the asymmetries. 